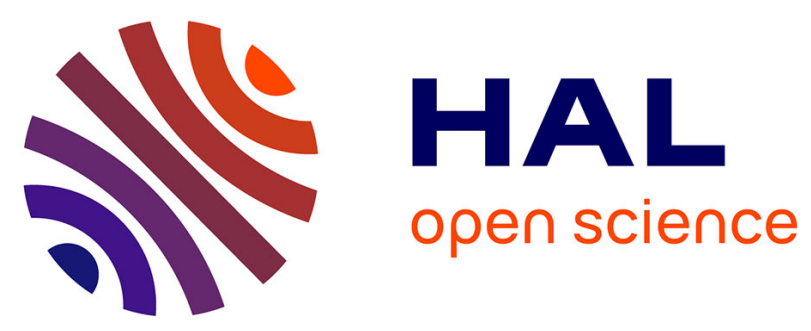

\title{
Kinetic scheme for arterial and venous blood flow, and application to partial hepatectomy modeling.
}

\author{
Chloe Audebert, Petru Bucur, Mohamed Bekheit, Eric Vibert, Irene
}

Vignon-Clementel, Jean-Frédéric Gerbeau

\section{To cite this version:}

Chloe Audebert, Petru Bucur, Mohamed Bekheit, Eric Vibert, Irene Vignon-Clementel, et al.. Kinetic scheme for arterial and venous blood flow, and application to partial hepatectomy modeling.. Computer Methods in Applied Mechanics and Engineering, 2017, 314, pp.102-125. 10.1016/j.cma.2016.07.009 . hal-01347500v2

HAL Id: hal-01347500

https://hal.science/hal-01347500v2

Submitted on 6 Oct 2016

HAL is a multi-disciplinary open access archive for the deposit and dissemination of scientific research documents, whether they are published or not. The documents may come from teaching and research institutions in France or abroad, or from public or private research centers.
L'archive ouverte pluridisciplinaire HAL, est destinée au dépôt et à la diffusion de documents scientifiques de niveau recherche, publiés ou non, émanant des établissements d'enseignement et de recherche français ou étrangers, des laboratoires publics ou privés. 


\title{
Kinetic scheme for arterial and venous blood flow, and application to partial hepatectomy modeling.
}

\author{
Chloe Audebert ${ }^{\mathrm{a}, \mathrm{b}, * *}$, Petru Bucur ${ }^{\mathrm{c}, \mathrm{d}}$, Mohamed Bekheit ${ }^{\mathrm{c}, \mathrm{e}}$, Eric Vibert ${ }^{\mathrm{c}, \mathrm{e}}$, \\ Irene E. Vignon-Clementel ${ }^{\mathrm{a}, \mathrm{b}, *}$, Jean-Frédéric Gerbeau ${ }^{\mathrm{a}, \mathrm{b}, *}$ \\ ${ }^{a}$ Inria Paris, 2 rue Simone Iff, CS 42112, 75589 Paris Cedex 12, France \\ ${ }^{b}$ Sorbonne Universités UPMC Univ Paris 6, Laboratoire Jacques-Louis Lions, 4 Place \\ Jussieu, 75252 Paris cedex 05, France \\ ${ }^{c}$ Inserm Unité 1193, Villejuif, France \\ ${ }^{d}$ CHRU, hôpitaux de Tours, Chirurgie Hépato-biliaire et Pancréatique, Transplantation \\ Hépatique, Tours, France \\ ${ }^{e}$ AP-HP, Hôpital Paul Brousse, Centre Hépato-Biliaire, Villejuif, France
}

\begin{abstract}
The article introduces a kinetic scheme to solve the 1D Euler equations of hemodynamics, and presents comparisons of a closed-loop 1D-0D model with real measurements obtained after the hepatectomy of four pigs.

Several benchmark tests show that the kinetic scheme compares well with more standard schemes used in the literature, for both arterial and venous wall laws. In particular, it is shown that it has a good behavior when the section area of a vessel is close to zero, which is an important property for collapsible or clamped vessels. The application to liver surgery shows that a model of the global circulation, including $0 \mathrm{D}$ and $1 \mathrm{D}$ equations, is able to reproduce the change of waveforms observed after different levels of hepatectomy. This may contribute to a better understanding of the change of liver architecture induced by hepatectomy.
\end{abstract}

Keywords: Kinetic scheme; Arterial flow; Venous flow; Vessel collapse;

Surgery simulation.

\footnotetext{
A final version of this manuscript can be found in Computer Methods in Applied Mechanics and Engineering (2016)

*Authors contributed equally

** Corresponding author
}

Preprint submitted to Computer Methods in Applied Mechanics and EngineeringOctober 6, 2016 


\section{Introduction}

Liver partial ablation surgery, namely partial hepatectomy, is necessary to treat some pathologies. In order to get a functional regeneration of the liver, the weight ratio of the remaining liver to the body must be at least $0.5 \%$ for a healthy human [1]. However, the liver ablation percentage needs sometimes to be higher, in presence of large tumors for example. Post-operative liver failure may then occur due to insufficient functional liver mass.

When partial ablation is performed, the remaining liver experiences pressure and flow changes. The importance of the hemodynamics changes depends on ablation size, but their relationship remains unclear. Moreover, the remaining liver regeneration capacity seems to be impacted by the post-resection hemodynamics. A better understanding of the hemodynamics impact of hepatectomy might therefore help improve surgical practice. To contribute to this challenge, we adopt two approaches: one is based on animal experiments, the other on mathematical modeling and simulation. The present work shows that the simulations are able to reproduce, and possibly explain, some findings of the experiments.

Experiments have been performed on pigs. This species is a good animal model for our problem since its liver to body weight ratio is close to human's [2]. Pressure and flow in the main vessels of the liver have been recorded for different resection percentages. An interesting finding of these experiments was the following: at the resection time, waveform changes were observed repeatedly in the pressure and flow measured in the hepatic artery. These changes differ for $75 \%$ and $90 \%$ hepatectomy. Since it is hypothesized that there is a link between liver architecture and hemodynamics, and since liver architecture is important to understand liver regeneration, there is a strong interest in explaining these changes in pressure and flow waveforms.

A mathematical model able to reproduce this phenomenon must satisfy several requirements. First, it has to be able to capture wave propagation. A network of vessels modeled by systems of the one-dimensional (1D) hyperbolic Euler equations is a natural candidate in this respect. The liver being perfused 
by both arterial and venous blood, the model should be able to address both kinds of vessels. In addition, since during surgery some vessels can be clamped, the model and the numerical scheme should be able to handle the limit of vanishing cross-section area. In this work, we propose to use a kinetic scheme, in particular because of its interesting capability to preserve the positivity of the cross-section area. This scheme was originally developed for the Saint-Venant shallow water equations. To our knowledge, this is the first time that it is used to model collapsible vessels.

Second, keeping in mind that the liver receives about $25 \%$ of the cardiac output [3], hepatectomy may also influence the systemic circulation. It is therefore desirable to embed the network of 1D models within a closed-loop model of the whole circulation, including the liver. To keep a moderate complexity, this compartment can be treated with zero-dimensional (0D) models, also known as lumped-parameter models, i.e. governed by ordinary differential equations.

The paper is organized as follows. In Section 2, the hyperbolic equations are recalled and the kinetic scheme is described, along with the boundary and coupling conditions. The kinetic scheme is validated on benchmark cases, for both arterial and venous flows. In Section 3, the closed-loop 0D-1D model is presented and the effects of partial hepatectomy are studied numerically and compared with experimental observations. Section 4 ends the paper, with some conclusions and perspectives.

\section{Kinetic scheme for arterial and venous blood flow}

\subsection{The Euler equations of hemodynamics}

Blood flow in large vessels of the cardiovascular system can be represented with a collection of one-dimensional systems of nonlinear equations:

$$
\left\{\begin{aligned}
\partial_{t} A+\partial_{x}(A u) & =0 \\
\partial_{t}(A u)+\partial_{x}\left(\kappa A u^{2}\right)+\frac{A}{\rho} \partial_{x} p & =A g-f\left(A, A_{0}, u\right),
\end{aligned}\right.
$$

The first equation corresponds to mass conservation and the second to momentum conservation. $x \in \mathbb{R}$ denotes the coordinate along the longitudinal axis of 
the portion of vessel, $t \in \mathbb{R}^{+}$is the time, $A(x, t)$ is the vessel cross-section area, $u(x, t)$ is the mean velocity of blood through the corresponding cross-section, $\rho$ is the fluid density assumed constant, $g$ denotes the gravity along the longitudinal axis, $f\left(A, A_{0}, u\right)$ is a friction term, and $\kappa$ is a momentum-flux correction coefficient, assumed to be equal to 1 in this work.

These equations have been used by many authors (e.g. $[4,5,6,7,8,9,10$, $11,12,13,14]$ to only name a few). Many variants exist, for example in the treatment of dissipation, viscoelasticity, curvature, momentum-flux correction coefficient, etc. Here we choose the simplest form of these different components, adding complexity through gravity and dissipation as needed by the test cases. The mean pressure $p(x, t)$ in a cross-section is related to the cross-section area through an algebraic constitutive law:

$$
p(x, t)=P_{0}(x)+\psi\left(A(x, t), A_{0}(x), \beta(x)\right),
$$

where $A_{0}(x)$ is a reference area, $P_{0}(x)$ is the pressure when $A(x, t)$ is equal to $A_{0}(x), \beta(x)$ is a parameter representing the vessel stiffness, and $\psi$ is a given function characterizing the "tube law". The details regarding the tube law for this work can be found in $[7,15]$ for arteries, and in $[16,17,18,19]$ for veins. Eliminating the pressure from system (1) gives:

$$
\left\{\begin{aligned}
\partial_{t} A+\partial_{x}(A u) & =0 \\
\partial_{t}(A u)+\partial_{x}\left(A u^{2}\right)+\frac{A}{\rho}\left(\partial_{A} \psi\right) \partial_{x} A & =A g-f\left(A, A_{0}, u\right) \\
& -\frac{A}{\rho}\left(\partial_{x} P_{0}+\left(\partial_{A_{0}} \psi\right) \partial_{x} A_{0}+\left(\partial_{\beta} \psi\right) \partial_{x} \beta\right)
\end{aligned}\right.
$$

In order to write the system in conservative form, the term $\frac{A}{\rho}\left(\partial_{A} \psi\right) \partial_{x} A$ is reformulated:

$$
\frac{A}{\rho}\left(\partial_{A} \psi\right) \partial_{x} A=\frac{1}{\rho} \partial_{x}\left(\int_{\varepsilon A_{0}}^{A(x, t)} a \partial_{a} \psi\left(a, A_{0}, \beta\right) d a\right)=\partial_{x}\left(\int_{\varepsilon A_{0}}^{A(x, t)} c^{2}(a) d a\right)
$$

where $\varepsilon$ is a constant whose value will be discussed later, and $c(A)=\sqrt{\frac{A}{\rho} \partial_{A} \psi}$ 
is the wave speed. With this reformulation, system (2) becomes:

$$
\left\{\begin{aligned}
\partial_{t} A+\partial_{x}(A u) & =0 \\
\partial_{t}(A u)+\partial_{x}\left(A u^{2}\right)+\partial_{x}\left(\int_{\varepsilon A_{0}}^{A(x, t)} c^{2}(a) d a\right) & =A g-f\left(A, A_{0}, u\right)-\frac{A}{\rho}\left(\partial_{x} P_{0}\right. \\
& \left.+\left(\partial_{A_{0}} \psi\right) \partial_{x} A_{0}+\left(\partial_{\beta} \psi\right) \partial_{x} \beta\right)
\end{aligned}\right.
$$

When the reference cross-section area $A_{0}$, the stiffness parameter $\beta$ and the pressure $P_{0}$ are assumed to be constant in space, the system reads:

$$
\left\{\begin{aligned}
\partial_{t} A+\partial_{x}(A u) & =0 \\
\partial_{t}(A u)+\partial_{x}\left(A u^{2}\right)+\partial_{x}\left(\int_{\varepsilon A_{0}}^{A(x, t)} c^{2}(a) d a\right) & =A g-f\left(A, A_{0}, u\right) .
\end{aligned}\right.
$$

\subsection{The kinetic scheme}

Many numerical methods have been used in the literature to address the solution of (5). In the arterial case, we refer to the recent overview presented in [20], where six different methods were compared: discontinuous Galerkin, locally conservative Galerkin, Galerkin least-squares finite element, finite volume, finite difference MacCormack, and a simplified trapezium rule method (STM). In the venous case, a Godunov scheme has been used in [18], an ADER (Arbitrary Accuracy DERivative Riemann problem) scheme in [19], and a Runge-Kutta discontinuous Galerkin scheme in [21].

In the present work, a kinetic scheme is adopted for both arterial and venous flows. A motivation for this method, which was initially proposed for the SaintVenant equations [22], is its capability to provably preserve the positivity of the cross-section area, which is especially relevant in collapsible vessels. To our knowledge, this is the first time this scheme is used in hemodynamics for collapsible vessels. It was recently used for arterial flow in [23].

A kinetic interpretation of system (5) is obtained by introducing a linear microscopic kinetic equation equivalent to the macroscopic model [24]. A real function $\chi$ defined on $\mathbb{R}$ is introduced. It is compactly supported and verifies 
the following properties:

$$
\left\{\begin{array}{l}
\chi(-w)=\chi(w) \geq 0 \\
\int_{\mathbb{R}} \chi(w) d w=\int_{\mathbb{R}} w^{2} \chi(w) d w=1 .
\end{array}\right.
$$

Here, this function is defined by $\chi(w)=\frac{1}{2 \sqrt{3}} \mathbf{1}_{|w| \leq \sqrt{3}}$, but other choices are possible [24]. A distribution function $M(x, t, \xi)$ is introduced:

$$
M(x, t, \xi)=\frac{A}{\gamma} \chi\left(\frac{\xi-u}{\gamma}\right)
$$

with $\gamma^{2}=\frac{1}{A} \int_{\varepsilon A_{0}}^{A(x, t)} c^{2}(a) d a$. The wave speed $c$ will be further specified in the next section for arterial and venous blood flows. In the kinetic formalism, the variable $\xi$ represents the microscopic particle velocity.

Consider first the case without source terms. The equation verified by $M$ and the system (5) are linked with the following result [24]: the functions $A$ and $u$ are solutions to the Euler equations (5), if and only if $M(x, t, \xi)$ is solution to the kinetic equation

$$
\partial_{t} M+\xi \partial_{x} M=\mathcal{Q}(x, t, \xi),
$$

where $\mathcal{Q}(x, t, \xi)$ is a collision term that satisfies:

$$
\int_{\mathbb{R}} \mathcal{Q} d \xi=\int_{\mathbb{R}} \xi \mathcal{Q} d \xi=0
$$

The link between the microscopic density function and the macroscopic variable is given by the two relations:

$$
\int_{\mathbb{R}} M d \xi=A, \quad \int_{\mathbb{R}} \xi M d \xi=A u .
$$

Let $\Delta t$ and $\Delta x$ denote the time and space steps respectively. Let $\left(A_{i}^{n}, u_{i}^{n}\right)$ denote an approximation of $\left(A\left(x_{i}, t_{n}\right), u\left(x_{i}, t_{n}\right)\right)$, with $t_{n}=n \Delta t$ and $x_{i}=i \Delta x$. The unknown $\left(A_{i}^{n}, u_{i}^{n}\right)$ is solution to a finite volume kinetic scheme deduced from the kinetic interpretation of the equations. Let $M_{i}^{n}$ be the discrete particles density, defined by

$$
M_{i}^{n}=M_{i}^{n}(\xi)=\frac{A_{i}^{n}}{\gamma_{i}^{n}} \chi\left(\frac{\xi-u_{i}^{n}}{\gamma_{i}^{n}}\right)
$$


with $\gamma_{i}^{n}=\left(\frac{1}{A_{i}^{n}} \int_{\varepsilon A_{0}}^{A_{i}^{n}} c^{2}(a) d a\right)^{\frac{1}{2}}$. Equation (7) is approximated by an upwind scheme:

$$
M_{i}^{n+1,-}=M_{i}^{n}-\frac{\Delta t}{\Delta x} \xi\left(M_{i+\frac{1}{2}}^{n}-M_{i-\frac{1}{2}}^{n}\right),
$$

with $M_{i+\frac{1}{2}}^{n}=M_{i}^{n} \mathbf{1}_{\xi \geq 0}+M_{i+1}^{n} \mathbf{1}_{\xi \leq 0}$. Then $A_{i}^{n}$ and $(A u)_{i}^{n}=A_{i}^{n} u_{i}^{n}$ are computed with (8):

$$
X_{i}^{n+1}=\left(\begin{array}{l}
A_{i}^{n+1} \\
A_{i}^{n+1} u_{i}^{n+1}
\end{array}\right)=\int_{\mathbb{R}}\left(\begin{array}{l}
1 \\
\xi
\end{array}\right) M_{i}^{n+1,-} d \xi .
$$

The kinetic scheme reads:

$$
X_{i}^{n+1}=X_{i}^{n}-\frac{\Delta t}{\Delta x}\left(\mathcal{F}_{i+\frac{1}{2}}^{n}-\mathcal{F}_{i-\frac{1}{2}}^{n}\right),
$$

with $\mathcal{F}_{i+\frac{1}{2}}^{n}=\int_{\mathbb{R}} \xi\left(\begin{array}{l}1 \\ \xi\end{array}\right) M_{i+\frac{1}{2}}^{n} d \xi$. Given the function $\chi$ chosen above, the following integrals can be computed in closed form:

$$
\begin{aligned}
\int_{\xi \geq 0}\left[\xi^{p} \frac{A}{\gamma} \chi\left(\frac{\xi-u}{\gamma}\right)\right] d \xi & =\frac{1}{2 \sqrt{3}} \frac{A}{\gamma(p+1)}\left[(\xi \gamma+u)^{p+1}\right]_{\xi=\max \left(\frac{-u}{\gamma} ;-\sqrt{3}\right)}^{\xi=\max \left(\frac{-u}{\gamma}\right)} \quad p=1,2 \\
\int_{\xi \leq 0}\left[\xi^{p} \frac{A}{\gamma} \chi\left(\frac{\xi-u}{\gamma}\right)\right] d \xi & =\frac{1}{2 \sqrt{3}} \frac{A}{\gamma(p+1)}\left[(\xi \gamma+u)^{p+1}\right]_{\xi=\min \left(\frac{-u}{\gamma} ;-\sqrt{3}\right)}^{\xi=\min \left(\frac{-u}{\gamma}\right)}
\end{aligned}
$$

which gives the expression of the flux $\mathcal{F}$.

In presence of a source term, we adopt the simple strategy of an explicit treatment:

$$
X_{i}^{n+1}=X_{i}^{n}-\frac{\Delta t}{\Delta x}\left(\mathcal{F}_{i+\frac{1}{2}}^{n}-\mathcal{F}_{i-\frac{1}{2}}^{n}\right)+\Delta t^{n} S\left(X_{i}^{n}\right),
$$

where $S\left(X_{i}^{n}\right)=\left(0, g A_{i}^{n}-f\left(A_{i}^{n}, A_{0}, u_{i}^{n}\right)\right)^{T}$.

Under the CFL condition $\Delta t \max _{i}\left(\left|u_{i}^{n}\right|+\sqrt{3} \gamma_{i}^{n}\right) \leq \Delta x$, following the same arguments as in [22] for the shallow water equations, it can be proved that the scheme (12) preserves the positivity of the cross-section area, i.e $A_{i}^{n} \geq 0$, if this property holds at time zero. A second order extension of (12) can be obtained with standard arguments (minmod flux limiter).

Remark 1. As mentioned above, $A_{0}$ is assumed to be constant in each vessel. If $A_{0}$ was space-dependent, for example to account for the vessel tapering, 
the source term should be carefully treated to obtain a numerical scheme that ensures the equilibrium at rest. A similar issue was addressed for the shallow water equations in [25] with a technique called "hydrostatic reconstruction". To our knowledge, in the context of blood flow, this question was first addressed in [23] and named "dead man equilibrium". It was also addressed in [26] for a different scheme.

Arteries and veins tube laws. The tube law for arteries [15] is defined by,

$$
\psi\left(A, A_{0}, \beta_{a}\right)=\beta_{a}\left(\sqrt{A}-\sqrt{A_{0}}\right)
$$

with

$$
\beta_{a}=\frac{4 \sqrt{\pi} E h_{0}}{3 A_{0}},
$$

where $\mathrm{E}$ is the Young's modulus, and $h_{0}$ the thickness of the tube. The wave speed is then defined by:

$$
c^{2}=\frac{\beta_{a}}{2 \rho} \sqrt{A(x, t)}
$$

Thus, with the arterial tube law, $c^{2}$ is integrable in $A=0$ and we can choose $\varepsilon=0$ in (3). The kinetic distribution function $M$ is defined by $M(x, t, \xi)=$ $\frac{A}{\gamma} \chi\left(\frac{\xi-u}{\gamma}\right)$, with $\gamma^{2}=\frac{1}{A} \int_{0}^{A(x, t)} c^{2}(a) d a=\frac{\beta_{a}}{3 \rho} A^{\frac{1}{2}}=\frac{2}{3} c^{2}$ and system (5) reads:

$$
\left\{\begin{aligned}
\partial_{t} A+\partial_{x}(A u) & =0 \\
\partial_{t}(A u)+\partial_{x}\left(A u^{2}\right)+\partial_{x}\left(\frac{\beta}{3 \rho} A^{\frac{3}{2}}\right) & =A g-f\left(A, A_{0}, u\right),
\end{aligned}\right.
$$

with $p(x, t)=P_{0}+\beta_{a}\left(\sqrt{A(x, t)}-\sqrt{A_{0}}\right)$.

For collapsible tubes, like veins, we adopt the same tube law as in $[17,18,19]$ :

$$
\psi\left(A, A_{0}, \beta_{v}\right)=\beta_{v}\left(\left(\frac{A}{A_{0}}\right)^{m}-\left(\frac{A}{A_{0}}\right)^{n}\right),
$$

where $\beta_{v}$ is an elasticity parameter. With $m=10$ and $n=-1.5$, which are the values commonly used in the literature, the squared wave speed defined by

$$
c^{2}=\frac{\beta_{v}}{\rho}\left(m\left(\frac{A}{A_{0}}\right)^{m}-n\left(\frac{A}{A_{0}}\right)^{n}\right)
$$


is not integrable at $A=0$. This question would deserve a special study. Here, for simplicity, we circumvent this difficulty by taking $\epsilon>0$ in (3):

$$
\begin{aligned}
\gamma^{2} & =\frac{1}{A} \frac{\beta_{v}}{\rho} \int_{\varepsilon A_{0}}^{A(x, t)}\left(m\left(\frac{a}{A_{0}}\right)^{m}-n\left(\frac{a}{A_{0}}\right)^{n}\right) d a \\
& =\frac{\beta_{v}}{\rho}\left(\frac{m}{m+1}\left(\frac{A}{A_{0}}\right)^{m}-\frac{n}{n+1}\left(\frac{A}{A_{0}}\right)^{n}-\frac{A_{0}}{A}\left(\frac{m}{m+1} \varepsilon^{m+1}-\frac{n}{n+1} \varepsilon^{n+1}\right)\right) .
\end{aligned}
$$

The value of $\varepsilon$ will be discussed in section 2.4.

\subsection{Boundary treatments}

Characteristic variables. The characteristic variables are computed from the quasi-linear form of system (5). For arterial blood flow [15], the characteristic variables are:

$$
W_{+}=u+4 \sqrt{\frac{\beta}{2 \rho}} A^{\frac{1}{4}}, \quad W_{-}=u-4 \sqrt{\frac{\beta}{2 \rho}} A^{\frac{1}{4}} .
$$

For venous blood flow [19], the characteristic variables are:

$$
W_{+}=u+\int_{A_{0}}^{A} \frac{c(a)}{a} d a, \quad W_{-}=u-\int_{A_{0}}^{A} \frac{c(a)}{a} d a .
$$

In the following numerical examples, the characteristic variables are approximated with the trapezoidal rule for venous blood flow.

Transmission conditions. In presence of a bifurcation, or a change in material properties, conservation of mass is imposed: $Q_{m}=Q_{d_{1}}+Q_{d_{2}}$, where $Q=A u$ denotes the flow rate, $m$ the mother vessel and $d_{1}, d_{2}$ the two daughter vessels. Except in some specific cases detailed below, continuity of the total pressure $P_{T}=\frac{\rho}{2} u^{2}+p$ is also imposed: $P_{T, m}=P_{T, d_{1}}=P_{T, d_{2}}$. These relations are complemented with the relations provided by the outgoing characteristics, as explained e.g. in [15]. The resulting system of nonlinear equations is then solved with a Newton method.

Boundary conditions. Different types of boundary conditions are considered in the numerical examples. At the inlet of the open-loop models, either the pressure or the flow rate are imposed. At the outlet, either a constant pressure, or an 
absorbing boundary condition, or a coupling with a 0D model are used. For the absorbing boundary condition, the incoming characteristic variable is assumed constant in time. For the coupling with a 0D model, the differential equations are approximated with an implicit Euler scheme. Again, these relations are complemented with the information obtained from the outgoing characteristics. A Newton method and a parabolic linesearch algorithm are used to solve the resulting system of nonlinear equations.

\subsection{Benchmark test cases}

Arterial flow. Various benchmark test cases were proposed in [20] to compare six numerical methods for $1 \mathrm{D}$ blood flow models. Two representative tests are studied in the following paragraphs: a single pulse propagation, and an aortic bifurcation simulation. The kinetic scheme is compared to the results from [20]. For the two cases, the system (5) is solved, with a friction function defined by $f\left(A, A_{0}, u\right)=K_{f} u(x, t), K_{f}$ being constant, and gravity is neglected.

Single pulse propagation The first test case is the (non-physiological) propagation of a pulse wave along a tube, with an absorbing outlet boundary condition. Table 6 provides the parameters values. The inlet flow is imposed: $Q_{0}(t)=\exp \left(-10^{4}(t-0.05)^{2}\right) \mathrm{cm}^{3} \mathrm{~s}^{-1}$. First, the test is performed with the first order kinetic scheme, and the friction is neglected. In [20] all the numerical schemes give identical results for this benchmark. The kinetic scheme is here compared with the STM scheme. For $\Delta t=10^{-4} \mathrm{~s}$ and $\Delta x=10^{-1} \mathrm{~cm}$ the resulting pressure curves are shown in Figure 1 (blue curve). An excessive numerical diffusion is observed, which is reduced when space and time steps are refined (Figure 1 red curve), $\Delta t=10^{-5} \mathrm{~s}$ and $\Delta x=10^{-2} \mathrm{~cm}$ ). The results obtained with the second order in space kinetic scheme, with $\Delta t=10^{-5} \mathrm{~s}$ and $\Delta x=10^{-1} \mathrm{~cm}$, are plotted in Figure 2 (a). Figure 2 (b) shows the results obtained in the viscous case with the second order in space kinetic scheme $\left(\Delta t=10^{-5} \mathrm{~s}\right.$ and $\left.\Delta x=10^{-1} \mathrm{~cm}\right)$. Table 7 summarizes the normalized errors for all presented simulations. With the second order kinetic scheme, the results 


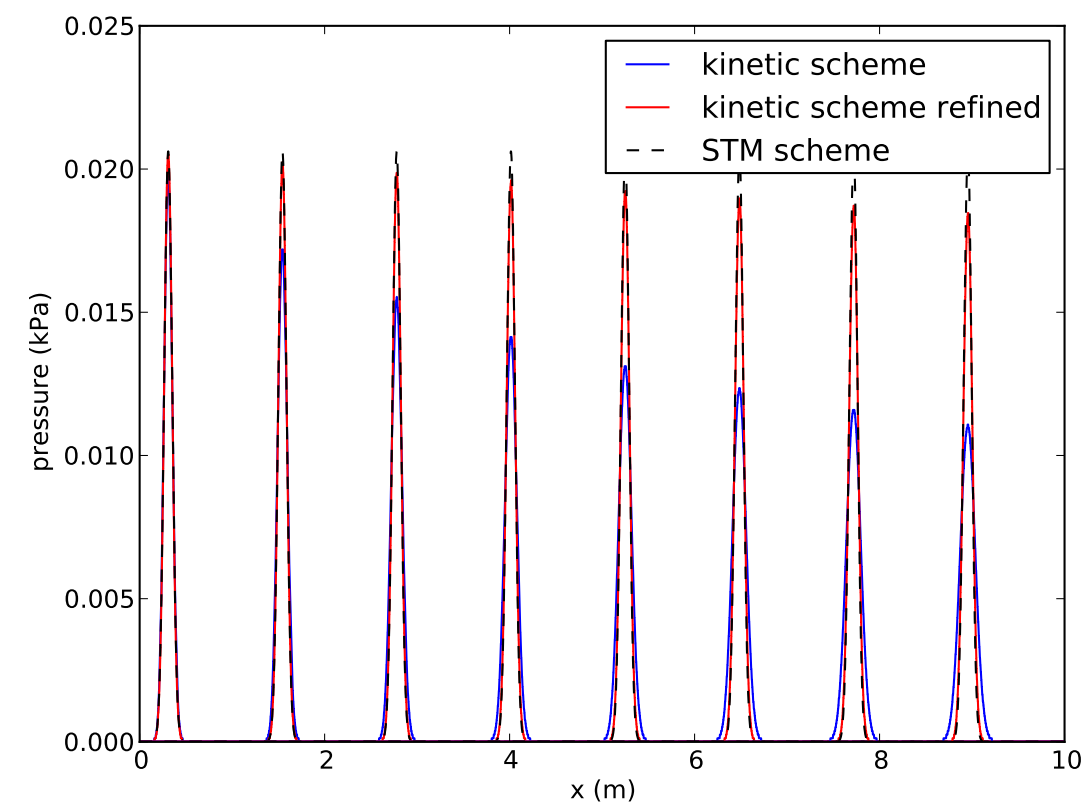

Figure 1: Comparison between time and space first order kinetic scheme results with $\Delta x=$ $0.1 \mathrm{~cm}$, and $\Delta t=1.010^{-4} \mathrm{~s}$ (blue), with $\Delta x=0.01 \mathrm{~cm}$, and $\Delta t=1.010^{-5} \mathrm{~s}$ (red) and numerical results from [20] (dash black) for the inviscid single pulse propagation test. The pressure over space is represented for different time instants: $0.1 \mathrm{~s}, 0.3 \mathrm{~s}, 0.5 \mathrm{~s}, 0.7 \mathrm{~s}, 0.9 \mathrm{~s}, 1.1 \mathrm{~s}, 1.3 \mathrm{~s}$, and $1.5 \mathrm{~s}$.

are in excellent agreement with the STM scheme.

Aortic bifurcation The second arterial test is an abdominal aorta branching into two symmetric iliac arteries. The vessel parameters are in Table 8. Two three-element Windkessel models represent the rest of the systemic circulation and are coupled to the two 1D iliac arteries. The flow rate is imposed at the inlet. Space and time steps for the kinetic scheme are $\Delta t=510^{-5} \mathrm{~s}$ and $\Delta x=0.1 \mathrm{~cm}$. The CFL number $\left(\Delta t \max _{i}\left(\left|u_{i}^{n}\right|+\sqrt{3} \gamma_{i}^{n}\right) / \Delta x\right)$ remains around 0.63. Figure 3 shows pressure, flow rate and radius change for the middle and the end points of the aorta, and the middle point of the iliac artery, compared to the results of $3 \mathrm{D}$ simulations and of the 1D scheme STM presented in [20]. 


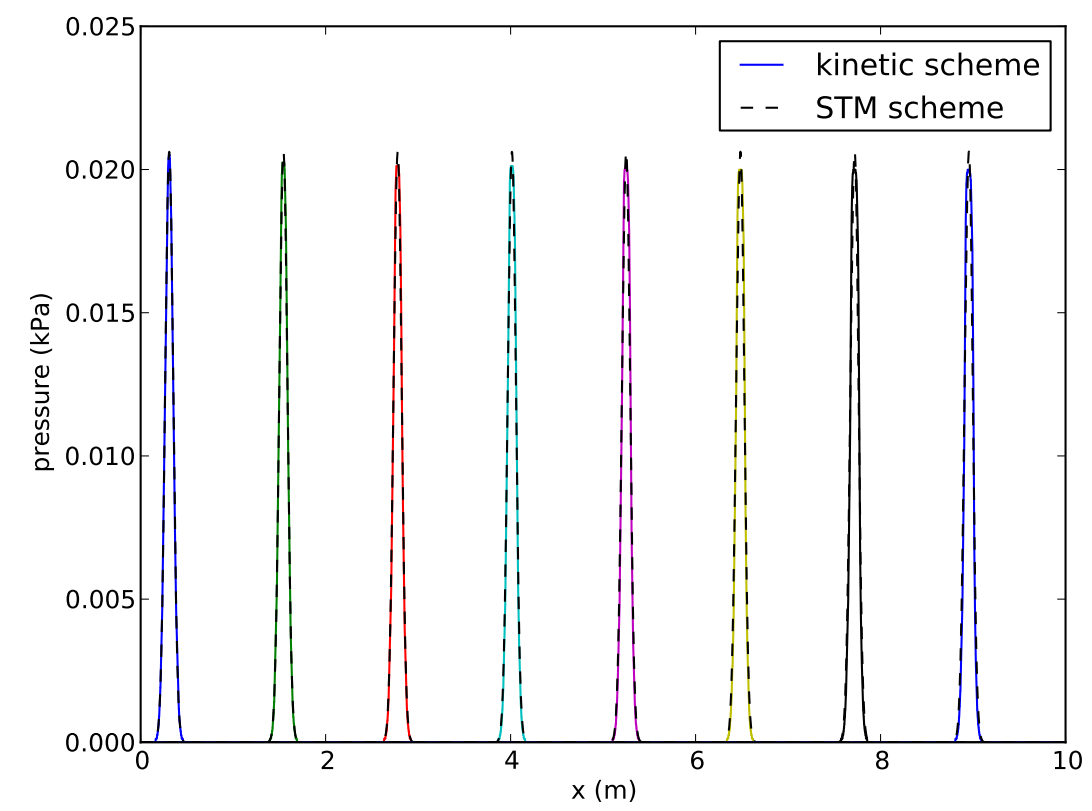

(a) Inviscid blood

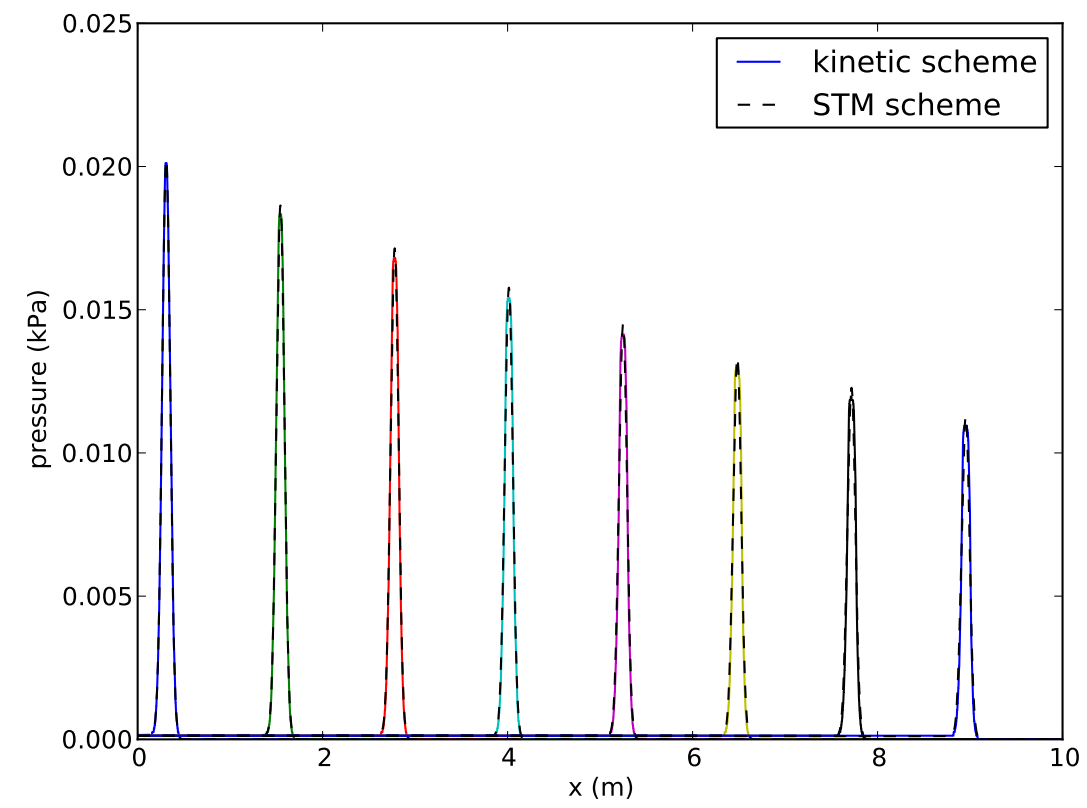

(b) Viscous blood

12

Figure 2: Comparison between first order in time and second order in space kinetic scheme results with $\Delta x=0.1 \mathrm{~cm}$, and $\Delta t=1.010^{-5} \mathrm{~s}$ and numerical results from [20] with inviscid (a) and viscous (b) blood for the single pulse propagation test. The pressure over space is represented for various times: $0.1 \mathrm{~s}, 0.3 \mathrm{~s}, 0.5 \mathrm{~s}, 0.7 \mathrm{~s}, 0.9 \mathrm{~s}, 1.1 \mathrm{~s}, 1.3 \mathrm{~s}$, and $1.5 \mathrm{~s}$. 
In [20] errors with respect to 3D solution are computed. The errors are defined by:

$$
\begin{gathered}
\mathcal{E}_{P}^{R M S}=\sqrt{\frac{1}{n} \sum_{i=1}^{n}\left(\frac{P_{i}-\mathcal{P}_{i}}{\mathcal{P}_{i}}\right)}, \quad \mathcal{E}_{Q}^{R M S}=\sqrt{\frac{1}{n} \sum_{i=1}^{n}\left(\frac{Q_{i}-\mathcal{Q}_{i}}{\max _{j}\left(\mathcal{Q}_{j}\right)}\right)} \\
\mathcal{E}_{P}^{M A X}=\max _{i}\left|\frac{P_{i}-\mathcal{P}_{i}}{\mathcal{P}_{i}}\right|, \quad \mathcal{E}_{Q}^{M A X}=\max _{i}\left|\frac{Q_{i}-\mathcal{Q}_{i}}{\max _{j}\left(\mathcal{Q}_{j}\right)}\right| \\
\mathcal{E}_{P}^{S Y S}=\frac{\max (P)-\max (\mathcal{P})}{\max (\mathcal{P})}, \quad \mathcal{E}_{Q}^{S Y S}=\frac{\max (Q)-\max (\mathcal{Q})}{\max (\mathcal{Q})} \\
\mathcal{E}_{P}^{D I A S}=\frac{\min (P)-\min (\mathcal{P})}{\min (\mathcal{P})}, \quad \mathcal{E}_{Q}^{D I A S}=\frac{\min (Q)-\min (\mathcal{Q})}{\max (\mathcal{Q})},
\end{gathered}
$$

where $P_{i}$ and $Q_{i}$ are the $1 \mathrm{D}$ simulation results at a given space point $x_{i}, \quad i \in$ $1 \ldots N, \mathcal{P}_{i}$ and $\mathcal{Q}_{i}$ are the $3 \mathrm{D}$ solutions at the same space location. The errors for $\Delta P$ and $\Delta r$ are defined similarly. Table 9 presents the errors. Again, the kinetic scheme is in very good agreement with the other schemes presented in [20]. In that case, which is more physiological than the previous one, the first order kinetic scheme is sufficient to reach a good accuracy with reasonable discretization steps.

Venous flow. After having been tested on arterial benchmarks, the kinetic scheme is now applied to venous flow, which is more challenging. For collapsible tubes, such as veins, the squared speed wave (eq. 18) is not integrable at $A=0$. This difficulty is avoided by taking $\varepsilon>0$ in (3). In the following numerical simulations, we took the value $\varepsilon=10^{-3}$. We noticed that the solution was slightly sensitive to the value of $\varepsilon$, but this dependency is reduced when space and time steps are decreased.

Jugular vein collapse The test of the "giraffe jugular vein" was proposed in $[17,18]$, and used more recently in $[21,19]$. A single vein is considered with length $L=200 \mathrm{~cm}$, cross-section area $A_{0}=5 \mathrm{~cm}^{2}$ and material property parameter $\beta_{v}=50 \mathrm{dyn} / \mathrm{cm}^{2}$. A constant flow rate is imposed at the inlet and a fixed cross-section area at the outlet. The value of gravity is $g=980.0 \mathrm{~cm} / \mathrm{s}^{2}$, 

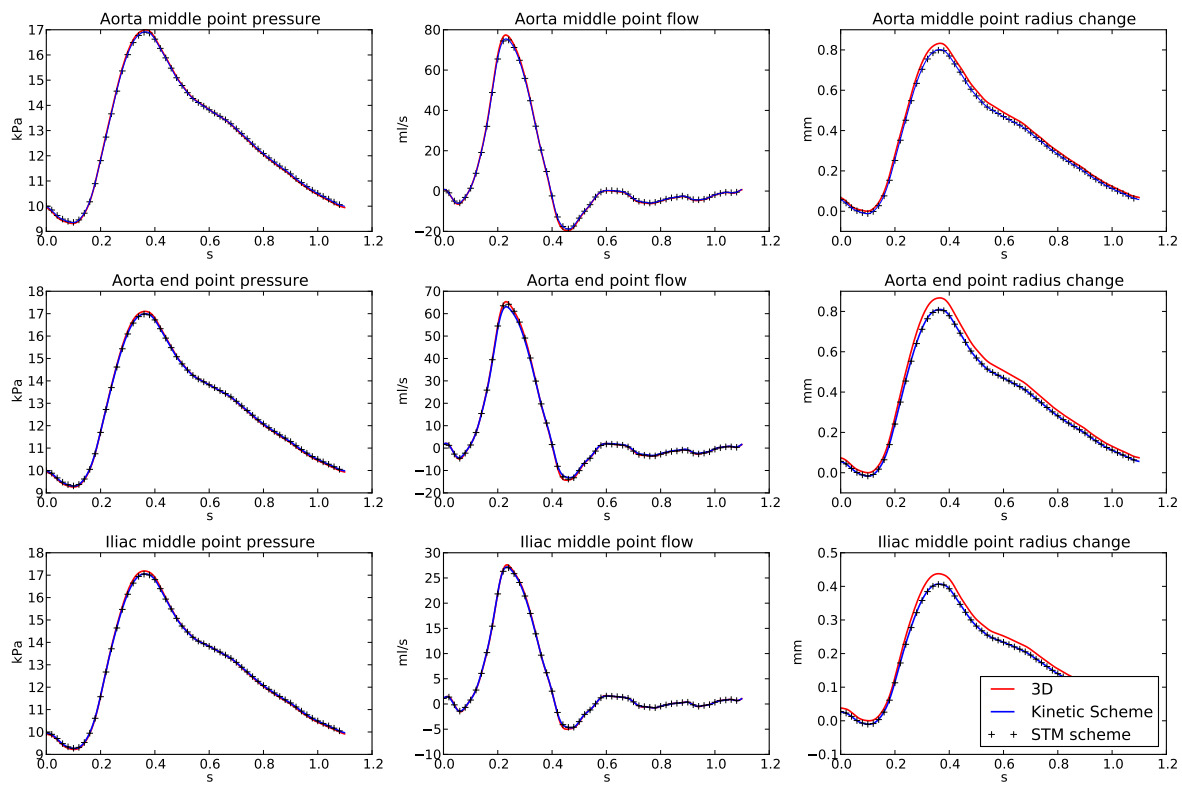

Figure 3: Aorta bifurcation test case: kinetic first order scheme results with $\Delta t=5.10^{-5} \mathrm{~s}$ and $\Delta x=0.1 \mathrm{~cm}$, and 3D and STM scheme results from [20], over one cardiac cycle. 
and the friction is defined by $f\left(A, A_{0}, u\right)=K_{f} \sqrt{\frac{A(t, x)}{A_{0}}} u(t, x)$, with $K_{f}=0.96$ $\mathrm{cm}^{2} / \mathrm{s}$. The initial conditions are $A(x, 0)=A_{0}\left(0.2+1.8 \frac{x}{L}\right)$ and $u(x, 0) A(x, 0)=$ $40 \mathrm{~cm}^{3} / \mathrm{s}$. The boundary conditions are $u(0, t) A(0, t)=40 \mathrm{~cm}^{3} / \mathrm{s}$ and $A(L, t)=$ $2 A_{0}$. In case of a super-critical inlet, $u(0, t) A(0, t)=40 \mathrm{~cm}^{3} / \mathrm{s}$ and $A(0, t)=$ $0.3825 \mathrm{~cm}^{2}$ are the two imposed conditions. The system is solved with the first order kinetic scheme with $\Delta x=1.0 \mathrm{~cm}$ and $\Delta t=10^{-4} \mathrm{~s}$. The CFL number $\left(\Delta t \max _{i}\left(\left|u_{i}^{n}\right|+\sqrt{3} \gamma_{i}^{n}\right) / \Delta x\right)$ is around 0.06 for the chosen time and space steps. Here, 201 nodes are used to solve the problem, whereas 1000 nodes were used for the Godunov scheme in [18].

The results for $\alpha=A / A_{0}$ and the velocity $u$ are plotted over the vessel length for $t=5.7 \mathrm{~s}$ and $t=50 \mathrm{~s}$ in Figure 4 . Gravity tends to empty the upstream part of the vessel, thus a super-critical flow appears at the inlet. The vessel cross-section area at the outlet is forced to remain equal to $2 A_{0}$, hence the flow remains sub-critical at the outlet and a shock appears in the middle of the vessel. The position of the shock oscillates until the solution converges. Figure 4 shows the solution at time $t=5.7 \mathrm{~s}$ and the stationary state (time $t=50 \mathrm{~s}$ ). The obtained curves are very similar to the curves reported in $[19,21,18]$. The front position is $x / L=0.8$ in [18], $x / L=0.72$ in [21], $x / L=0.74$ in [19]. With our numerical scheme, the front position is $x / L=0.74$.

Portal vein uncollapse To illustrate the robustness of the kinetic scheme, we propose a new benchmark test case mimicking the uncollapse of the portal vein. During the surgery described in the following section, the surgeons clamp the main vessels perfusing the organ to avoid blood loss. When the clamp is in place, the vessel is collapsed. Once sutures are done, they remove the clamp. The proposed test is mimicking the uncollapse of the portal vein, just after the clamp removal.

We assume that a cross-section area of $0.5 \%$ of $A_{0}$ corresponds to a collapsed vessel. The 1D blood flow equations (5) are solved with the vein constitutive law (18) in a single vessel, with length $L=6 \mathrm{~cm}$, cross-section area $A_{0}=0.8 \mathrm{~cm}^{2}$ 

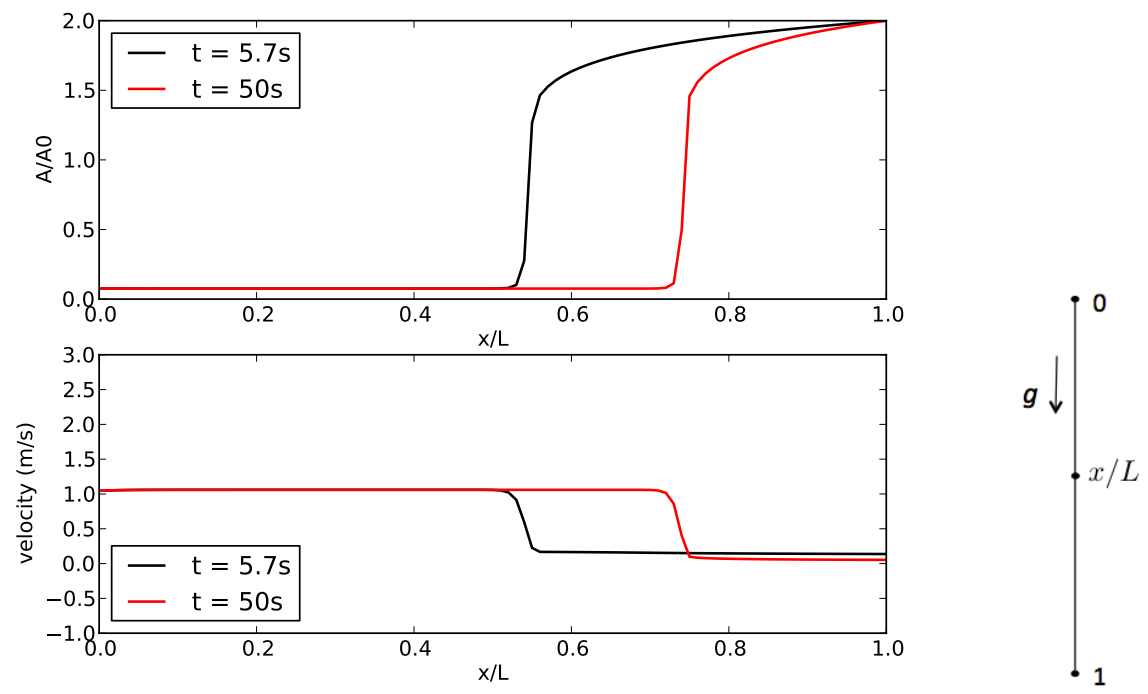

(a)

(b)

Figure 4: Giraffe jugular vein test: (a) at time $\mathrm{t}=5.7 \mathrm{~s}$ (black) and $\mathrm{t}=50 \mathrm{~s}$ (red), simulation results with kinetic scheme $\left(\Delta t=10^{-4} \mathrm{~s}, \Delta x=1.0 \mathrm{~cm}\right.$ ). $\alpha=\frac{A}{A_{0}}$ (top) and velocity in $\mathrm{m} / \mathrm{s}$ (bottom) are plotted over the vessel length. (b) schematic representation including gravity. 
and material parameter $\beta_{v}=10 \mathrm{dyn} / \mathrm{cm}^{2}$. The initial conditions are set to represent a collapsed vessel due to the clamp in the middle of the tube:

$$
\begin{aligned}
& A(x, 0)=\left(1.1-x \frac{10.95}{4 L}\right) A_{0} \text { for } x<4 L / 10 \\
& A(x, 0)=0.005 A_{0} \text { for } 4 L / 10 \leq x \leq 6 L / 10 \\
& A(x, 0)=\left(-1.6375+x \frac{10.95}{4 L}\right) A_{0} \text { for } 6 L / 10<x \leq L \\
& A(x, 0) u(x, 0)=0 \mathrm{~cm}^{3} / \mathrm{s}
\end{aligned}
$$

The inlet and outlet pressures are imposed, corresponding to a cross-section area of $1.1 A_{0}$. The pressure corresponding to $A=A_{0}$ is $P_{0}=1.05 \mathrm{mmHg}$, the blood density is $\rho=1 \mathrm{~g} \cdot \mathrm{cm}^{-3}$, the friction term is $f\left(A, A_{0}, u\right)=K_{f} u(t, x)$, with $K_{f}$ $=0.96 \mathrm{~cm}^{2} / \mathrm{s}$. The gravity is neglected. The first order kinetic scheme is used, with $\Delta x=0.05 \mathrm{~cm}$ and $\Delta t=10^{-4} \mathrm{~s}$. Figure 5 shows the quantity $\alpha=A / A_{0}$ for various time instants. At time $t=0 \mathrm{~s}$, just after unclamping, the vessel is collapsed in the middle. Then, the vessel uncollapses and oscillates around the equilibrium position (see $t=2 \mathrm{~s}$ and $t=5 \mathrm{~s}$ in Fig. 5 ) to eventually reach a steady state (see $t=20 \mathrm{~s}$ in Fig. 5 ).

\section{Application to hepatectomy}

To our knowledge, only a few mathematical models were proposed in the literature to describe the hemodynamics impact of liver surgeries. In [27], a cast-based reconstruction of the rat liver vasculature was performed to compute the resistance in the different vascular trees. Various sizes of virtual resection were studied with a resistance model and two $90 \%$ resection techniques were compared. The results indicated a portal hyperperfusion after resection and demonstrated that probably better outcomes could be expected with one of the two resection techniques. In [28], a 3D simulation was performed in the portal vein after right lobe hepatectomy. The geometry, based on medical imaging data, included superior mesenteric and splenic veins merging in portal vein and three portal vein branches. Constant velocities boundary conditions were prescribed in the mesenteric and splenic veins and zero pressure was imposed at 


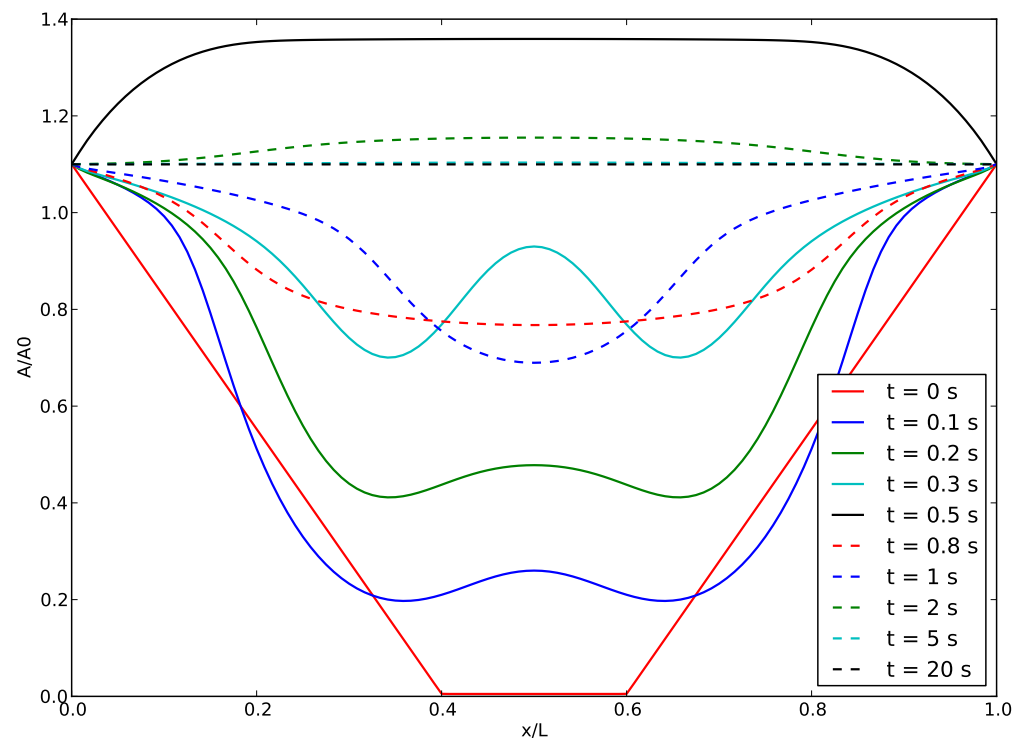

Figure 5: Uncollapse of portal vein simulation: $\alpha=\frac{A}{A_{0}}$ is plotted along the vessel at various time instants. 
the outlets. The right lobe hepatectomy was simulated changing the geometry. Similarly, for a two-lobe liver 0D model, driving conditions were kept unchanged before and after hepatectomy.

The model proposed in the present work differs from these two approaches from several aspects that will be detailed below. Our strategy is to propose a model of moderate complexity which can be parameterized to match measurements, but with a sufficient level of realism to be able to capture non-trivial phenomena observed in animal experiments.

\subsection{A closed-loop model}

To be able to consider waveform changes as a result - and not as an input - of the simulations, a closed-loop model is proposed including 1D and 0D compartments. Although this approach is not new, models of that sort, calibrated with experimental measurements, are not numerous in the literature. Closed-loop models, including 0D-1D-3D vessels, were proposed in [29] to study the impact of aortic insufficiency on the local hemodynamics of a cerebral aneurysm, and in [30] to study the effects of arterial and aortic valvular stenoses. Closed-loop 0D-1D models, including arteries and veins, were proposed in [31, 32]. The latter article focused on head and neck, to study possible connections between the venous vasculature and a class of neurodegenerative diseases. The simulation results were compared to Phase-Contrast MRI flow data.

The closed-loop model proposed in this work is represented in Figure 6. The main arteries are modeled with the 1D Euler equations described above. The arterial and venous trees at each outlet, as well as the pulmonary circulation and vena cava, are modeled with three-element Windkessel models. The heart and the liver are also represented by 0D models. The main features of each of these compartments are now detailed.

For the 1D models, the length, the cross-section area $A_{0}$ and the bifurcation angles are estimated from CT-scans of the pigs which underwent the surgical operations (CT-scans were done with a Siemens Somatom AS definition 128 machine). At the bifurcation, the continuity of total pressure is enforced as 


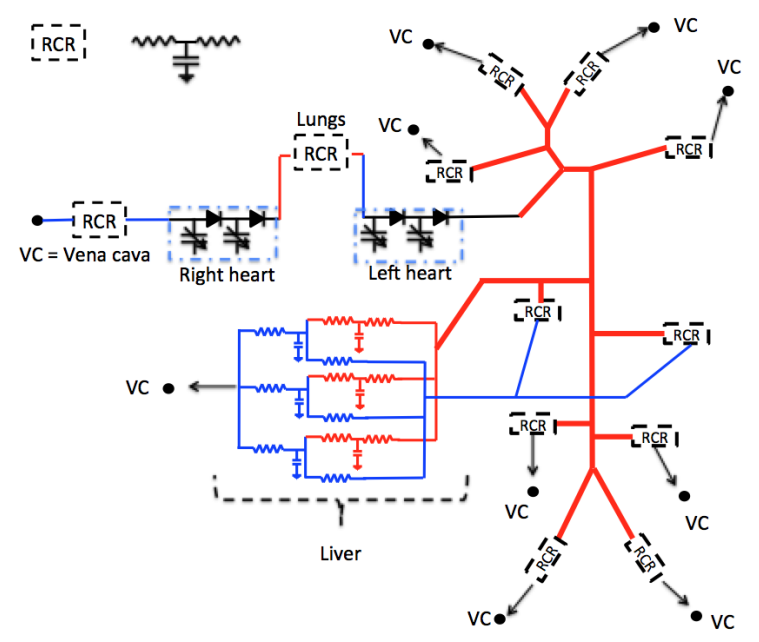

Figure 6: Schematic representation of the 1D-0D closed-loop model. 1D blood flow is simulated in the thick lines arteries while thin lines represent the OD model connections. All RCR units and the liver are linked (thin arrows) to the vena cava (VC).

explained in Section 2, except at the bifurcation between the abdominal aorta and the celiac trunk, and when the celiac trunk bifurcates into the hepatic and the splenic arteries. In these two bifurcations, the condition proposed in [7] is adopted: $P_{T, d_{1}}=P_{T, m}-2 \operatorname{sign}\left(u_{d_{1}}\right) u_{d_{1}}^{2} \sqrt{2\left(1-\cos \left(\alpha_{1}\right)\right.}$, where $\alpha_{1}$ is the angle of the branches $d_{1}$ with respect to the mother vessel.

The elasticity parameters are computed with the following formula [6]:

$$
\frac{E h_{0}}{r_{0}}=a \exp \left(b r_{0}\right)+c
$$

where $\mathrm{E}$ is the Young's modulus, $h_{0}$ and $r_{0}$ are the vessel thickness and radius when $A=A_{0}$. This formula is scaled in order to obtain a pressure in the carotid artery which is similar to the one measured in pigs. The parameters are: $a=6.010^{6} \mathrm{~g} \cdot \mathrm{s}^{-2} \cdot \mathrm{cm}^{-1}, b=-22.53 \mathrm{~cm}^{-1}$ and $c=2.59510^{5} \mathrm{~g} \cdot \mathrm{s}^{-2} \cdot \mathrm{cm}^{-1}$. The values for each artery can be found in Table 2 .

The total resistances for RCRs are computed with $R_{t o t}=\frac{\Delta P}{Q}$, by a combination of flow splits from [33], assuming pig and human flow splits are similar, and of available pressure and flow measurements. These total resistances are 
then separated into proximal $R_{p}$ and distal $R_{d}$ resistances, assuming the proximal resistance carries $10 \%$ of the total resistance in each RCR, within the ranges used in $[34,35,36]$. The total systemic capacitance is fixed at $4 \cdot 10^{-4} \mathrm{~cm}^{5} \cdot \mathrm{dyn}^{-1}$ as reported in [37] for pig circulation, further split based on the number of large arteries represented by each compartment according to [38]. Table 3 summarizes RCR parameter values.

The liver model is based on the pig anatomy. The pig liver consists of three separate lobes and is perfused by venous blood, through the portal vein, and arterial blood, through the hepatic artery. The three lobes are represented by three 0D models in parallel, connected to the heart through the 1D models, and to the digestive organs through the venous input connected to the RCR models of the splenic and mesenteric arteries. The vascular tree sizes are assumed proportional to the perfused tissue mass. A larger vascular tree has a smaller resistance, therefore the lobar resistances of the hepatic artery tree, the portal vein tree and the liver tissue are assumed inversely proportional to the lobe mass. The lobar capacitances of the hepatic artery tree and the liver tissue are assumed proportional to the lobe mass. The proximal to total resistance ratios of the hepatic artery tree reflect the lobar architecture differences [27]. The values of the liver parameters are reported in Table 4.

The functions governing the heart contraction come from the literature [39, 40, 30, 29], but their parameters are adapted to the pig heart. Heart valves are modeled with logistic functions, in order to obtain smooth yet sharp transitions between open and closed states. The heart chamber equations read:

$$
\left\{\begin{aligned}
\frac{d V_{i}}{d t} & =Q_{\text {in }, i}-Q_{\text {out }, i} \\
P_{i} & =E_{i}(t)\left(V_{i}-V_{0_{i}}\right) \\
Q_{\text {out }, i} & =G_{i}\left(P_{i}-P_{\text {out }, i}\right)\left(P_{i}-P_{\text {out }, i}\right)
\end{aligned}\right.
$$

where $i$ denotes either the right atrium (RA), right ventricle (RV), left atrium (LA) or left ventricle (LV); $V_{i}$ and $V_{0_{i}}$ are respectively the volume and unloaded volume of the heart chamber $i ; Q_{i n, i}$ and $Q_{o u t, i}$ are the incoming and outgoing flows; $P_{i}$ is the heart chamber pressure; $P_{i}-P_{\text {out }, i}$ is the pressure across the 
valve; $E_{i}$ is the elastance function, defined by $E_{i}(t)=E_{a_{i}} e(t)^{\alpha_{i}}+E_{b_{i}}$ with $\alpha_{i}=1$ if $i=R A, L A$ and $\alpha_{i}=0.5$ if $i=R V, L V$, as in [41]. $E_{a_{i}}$ and $E_{b_{i}}$ are the amplitude and baseline elastances respectively, and $e$ is a normalized time-varying function of the elastance, defined as follows for the ventricles:

$$
e(t)= \begin{cases}\frac{1}{2}\left[1-\cos \left(\pi \frac{t}{T_{v c}}\right)\right] & 0 \leq t \leq T_{v c} \\ \frac{1}{2}\left[1+\cos \left(\pi \frac{t-T_{v c}}{T_{v r}}\right)\right] & T_{v c}<t \leq T_{v c}+T_{v r} \\ 0 & T_{v c}+T_{v r}<t \leq T_{c c}\end{cases}
$$

and for the atria:

$$
e(t)= \begin{cases}\frac{1}{2}\left[1+\cos \left(\pi \frac{t+T_{c c}-t_{a r}}{T_{a r}}\right)\right] & 0 \leq t \leq t_{a r}+T_{a r}-T_{c c} \\ 0 & t_{a r}+T_{a r}-T_{c c}<t \leq t_{a c} \\ \frac{1}{2}\left[1-\cos \left(\pi \frac{t-t_{a c}}{T_{a c}}\right)\right] & t_{a c}<t \leq t_{a c}+T_{a c} \\ \frac{1}{2}\left[1+\cos \left(\pi \frac{t-t_{a r}}{T_{a r}}\right)\right] & t_{a c}+T_{a c}<t \leq T_{c c},\end{cases}
$$

where $T_{c c}$ is the duration of the cardiac cycle. The durations of the ventricular and atrial contractions and relaxations are denoted by $T_{v c}, T_{a c}, T_{v r}$ and $T_{a r}$ respectively; $t_{a c}$ and $t_{a r}$ are the times when the atria begin to contract and relax, respectively. The heart parameter values are given in Table 1 . The valve conductance is described by the function $G(\Delta P)=\frac{G_{0}}{1+\exp (-(\Delta P-d))}$ where $G_{0}=0.1 \mathrm{~cm}^{5} \cdot \mathrm{dyn}^{-1} \cdot \mathrm{s}^{-1}$, and $d=0.1 \mathrm{dyn} / \mathrm{cm}^{2}$.

Hepatectomy simulation in the $1 D-0 D$ closed loop model. The system of equations (5) is solved for the large arteries, with the first order kinetic scheme, with $\Delta x=0.1 \mathrm{~cm}$ and $\Delta t=10^{-4} \mathrm{~s}$ (see Table 5 ). Gravity is neglected and the friction function is defined as $f\left(A, A_{0}, u\right)=K_{f} u(x, t)$, with $K_{f}=3 \mathrm{~cm}^{2} / \mathrm{s}$. The initial conditions are $p(x, t=0)=45 \mathrm{mmHg}$ and $u(x, t=0)=5.0 \mathrm{~cm} / \mathrm{s}$.

Before hepatectomy, the 1D-0D closed loop model is tuned with the available measurements. Given the variability between subjects, the parameters are not tuned to represent a specific animal but to obtain representative pressures and flow rates. Figure 7 shows the measured carotid pressure curves over time for the four animals and the simulated curve. The first cardiac cycles at the left 
hand side of Figure 10 show the pressure and flow rates in the hepatic artery, to be compared with the experimental measurements represented in Figure 8. Given the intersubject variability, we considered that we reached a qualitative and quantitative agreement sufficient for our purpose.

The influence of partial hepatectomy on these hepatic artery waveforms is then studied by simulating partial hepatectomy in the model. In pig surgery, partial ablation is done in two steps. In a first stage, two of the three liver lobes are removed, corresponding to approximately $75 \%$ ablation. Part of the remaining lobe is removed in a second step to reach a final ablation around $90 \%$. The percentages of ablation are based on initial liver volume. In the model, the first stage is taken into account by dynamically increasing the corresponding lobe resistances and decreasing the corresponding capacitances, to simulate the $75 \%$ hepatectomy:

$R(t)=\left\{\begin{array}{l}R \text { if } t<T_{75} \\ R \exp \left(5\left(t-T_{75}\right)\right) \text { otherwise }\end{array} C(t)=\left\{\begin{array}{l}C \text { if } t<T_{75} \\ C \exp \left(-5\left(t-T_{75}\right)\right) \text { otherwise }\end{array}\right.\right.$

where $T_{75}$ is the time instant of the clamping, and $\mathrm{R}$ and $\mathrm{C}$ are the values before clamping. Then, to simulate the second part of the surgery, the remaining lobe mass is decreased, simulating a larger ablation resulting in a $90 \%$ hepatectomy. The remaining lobe mass is given by:

$$
M(t)=\left\{\begin{array}{l}
M \text { if } t<T_{90} \\
M\left(1-r+\frac{r}{1+\exp \left(-5\left(t-T_{90}\right)\right)}\right) \text { otherwise },
\end{array}\right.
$$

where $M$ is the initial lobe mass, $T_{90}$ is the time of the second clamp, and $r$ is the percentage removed in the remaining lobe. The parameters for the simulated hepatectomies are given in Table 4.

Typical changes in waveform occurring at $75 \%$ hepatectomy have been observed in several animals; Figure 8 shows four examples. The pressure amplitude goes up (between 5 and $10 \mathrm{mmHg}$ ). For the flow rate, although there is some variability in the pre-hepatectomy shape, after the clamping two characteristic changes can be observed besides the mean value decrease: the first peak is 
sharper, meaning the second peak is lower than before hepatectomy, and diastolic flow is at low values for longer. By contrast, no major changes in waveform have been observed for $75 \%$ to $90 \%$ hepatectomy, as shown in the experimental curves for two different pigs in Figure 9. Apart from a small mean flow decrease, in some pigs such as pig 4 , the flow rate minimum that follows systole becomes lower than the flow in diastole.

The two hepatectomies are simulated one after the other with the 1D-0D model. Figure 10 shows the simulated pressure and flow rate in the hepatic artery. For the $75 \%$ hepatectomy, the increase of pressure is well captured by the model and the typical changes of the flow waveform are well reproduced. For the simulated $75 \%$ to $90 \%$ hepatectomy, the pressure does not change and a small decrease in the mean and minimum flow appear (Figure 10 (b)) as in the experimental curves (Figure 9). Thus, the 1D-0D model is in good agreement with the experimental observations before and after clamping, both for $75 \%$ and $75 \%$ to $90 \%$ hepatectomies.

\subsection{Discussion}

It is quite remarkable that the $1 \mathrm{D}-0 \mathrm{D}$ model can predict the pressure and flow rate waveform changes for both $75 \%$ and $75 \%$ to $90 \%$ hepatectomies. This may be an indication that the waveforms are related to the liver architecture. To further understand this link, parameter sensitivity analysis can help to explain the changes in pressure amplitude and in flow waveform during the $75 \%$ hepatectomy. Generalized sensitivity functions (GSFs) analysis help identify correlations between parameters and the distribution over time of the information on parameters contained into the model outputs. Generalized sensitivity functions definitions are given in Appendix 5.2. Details on sensitivity analysis can be found in $[42,43,44,45]$. By definition, a GSF starts at value zero and ends at value one. The increase in-between is not necessary monotonic; if important correlations between parameters exist oscillations occur. The time interval where the sharpest increase occurs is when most information on the parameter is contained into the model output. The GSF is computed before 


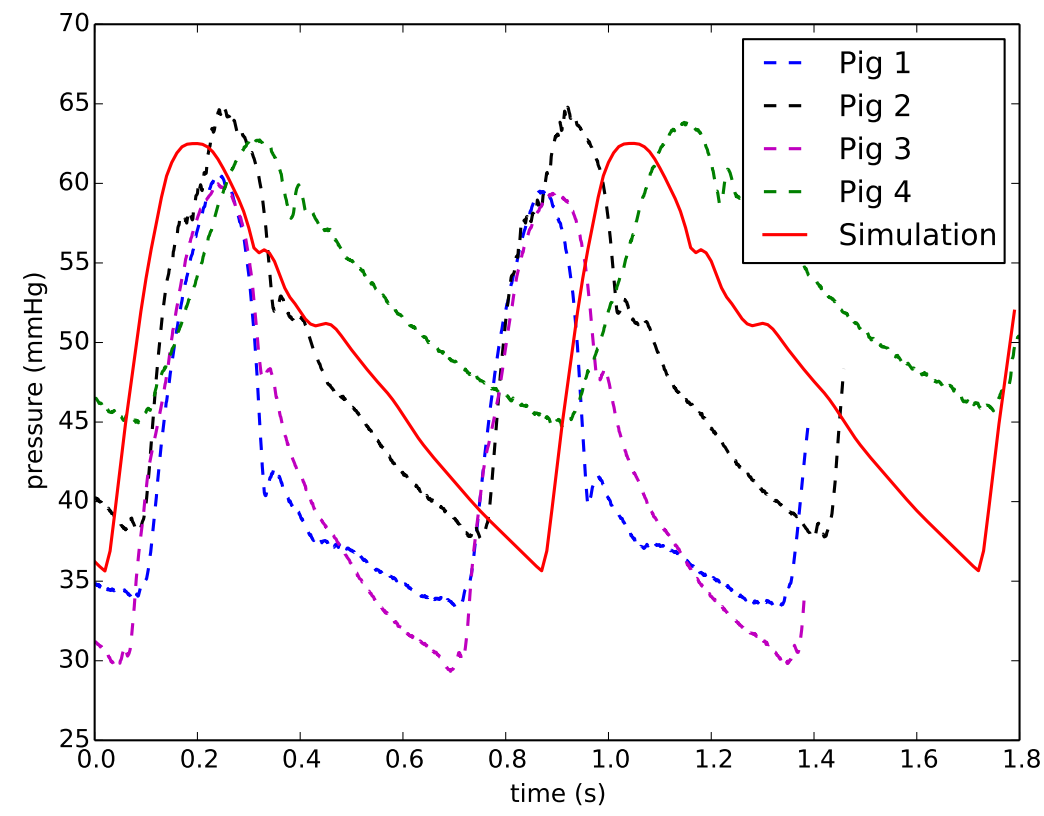

Figure 7: Carotid pressure over two cardiac cycles: measurement curves for four pigs (dashed lines) and 1D-0D closed-loop model simulated curve (solid line). 

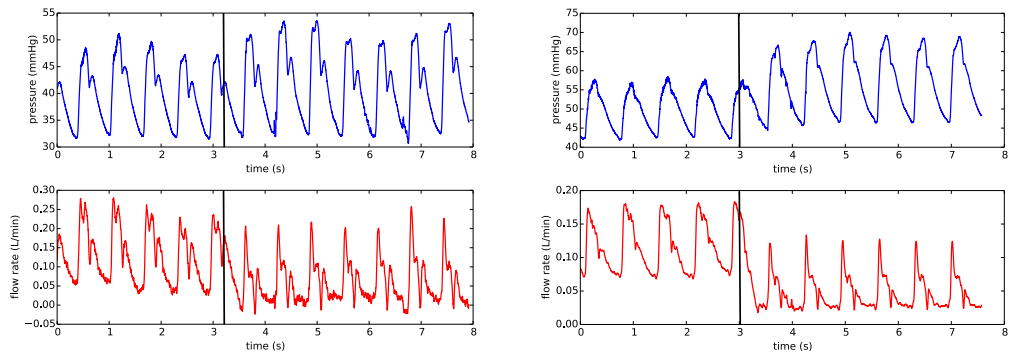

(a) Pig 1

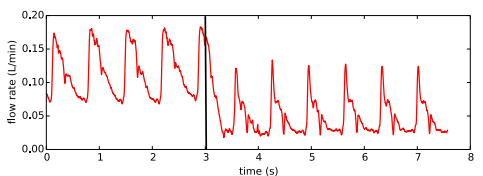

(b) Pig 2
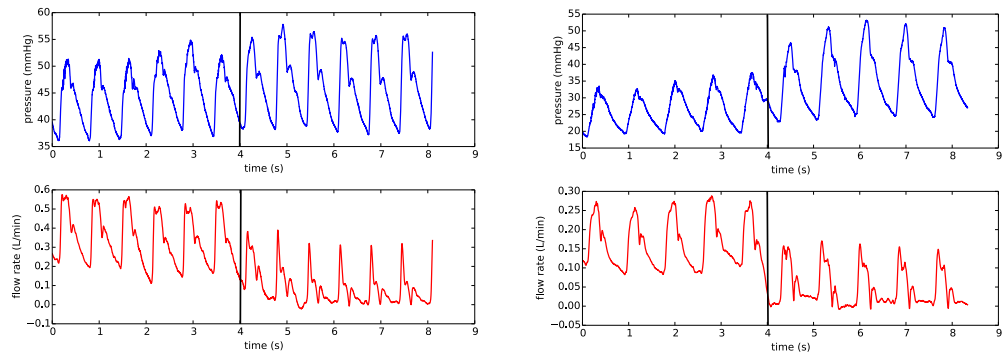

(c) Pig 3

(d) Pig 4

Figure 8: Experimental measurements of hepatic artery pressure and flow rate during $75 \%$ hepatectomy for four different pigs, the dark lines indicating the clamping time.
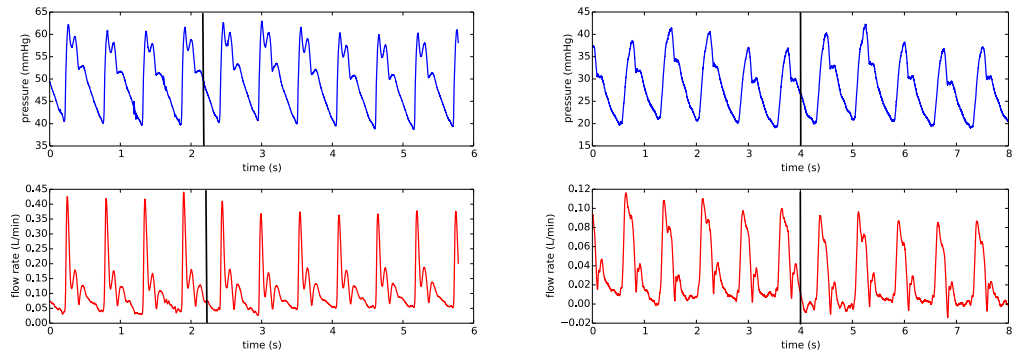

(a) Pig 3

(b) Pig 4

Figure 9: Experimental measurements of hepatic artery pressure and flow rate during $75 \%$ to $90 \%$ hepatectomy for two different pigs, the dark lines indicating the clamping time. 

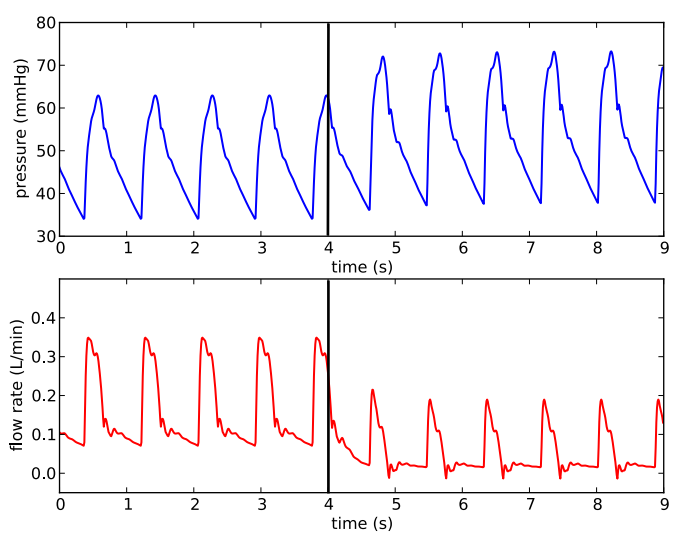

(a) $75 \%$ hepatectomy simulation
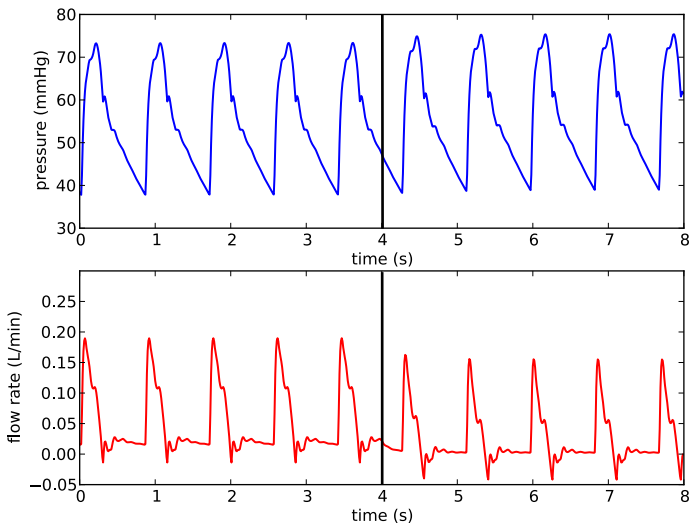

(b) $75 \%$ to $90 \%$ hepatectomy simulation

Figure 10: 1D-0D closed-loop model results: hepatic artery pressure $(\mathrm{mmHg})$ and flow rate (L/min) during $75 \%$ simulated hepatectomy and $75 \%$ to $90 \%$ simulated hepatectomy, the dark lines indicating the time of simulated clamping. 
and after the simulated $75 \%$ hepatectomy.

The GSFs of the total resistance and capacitance for flow and pressure in the hepatic arterial trees are plotted in Figures 11 and 12. Before hepatectomy, pressure and flow are sensitive to resistance during the entire cardiac cycle. This result is expected as resistance impacts mean pressure and flow. The pressure is more sensitive to the capacitance during its rising phase. The sharper increase of capacitance GSF after $75 \%$ hepatectomy indicates that the pressure amplitude is especially sensitive to capacitance. Between before and after $75 \%$ hepatectomy, capacitance is divided by approximately four. Before $75 \%$ hepatectomy, the flow is sensitive to resistance and capacitance during the entire cardiac cycle. After $75 \%$ hepatectomy, the flow is more sensitive to the capacitance between $0.3 \mathrm{~s}$ and $0.45 \mathrm{~s}$, corresponding to the sharp decrease in the flow curve.

Thus, the change in parameters due to the $75 \%$ hepatectomy - resistance increases by around $75 \%$ and capacitance decreases by around $75 \%$ - seems to explain the changes in pressure and flow waveforms. To confirm this hypothesis, the pre-hepatectomy model is run but with hepatic artery resistance and capacitance parameters multiplied and divided by four respectively, as if each lobe was $75 \%$ smaller. The new simulations are compared with the previous ones after the $75 \%$ hepatectomy, in Figure 13. Contrarily to pressures, the flow rates differ. Therefore, the change of global parameter values - total liver resistance and capacitance - can explain the change in pressure amplitude but it is not enough to obtain the sharp change observed in the flow waveform.

The fact that changes in hepatic artery flow waveform during experiments are observed for $75 \%$ hepatectomy but not for $90 \%$ hepatectomy, can be explained by the change in architecture in the blood vessel trees. Indeed, in the first hepatectomy, two of the three liver lobes are removed, which leads to an important architecture change. For the second hepatectomy, the remaining lobe mass is decreased and the vessel tree architecture does not change as much. In the model, the simulation of the first stage corresponds to an impedance change from 3 RCRs in parallel to a single RCR. For the second stage, the impedance remains the one of a single RCR; only the remaining lobe model parameters are 

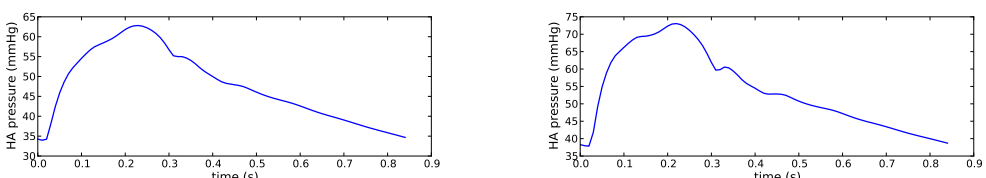

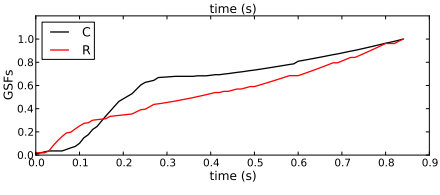

(a) Before partial hepatectomy

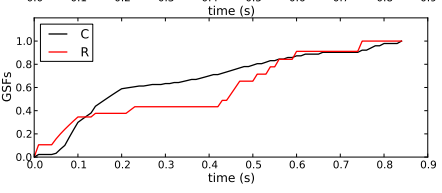

(b) After $75 \%$ hepatectomy

Figure 11: Pressure GSF of hepatic arterial trees for the resistance and the capacitance
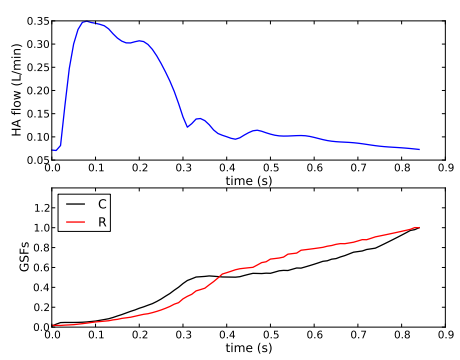

(a) Before partial hepatectomy
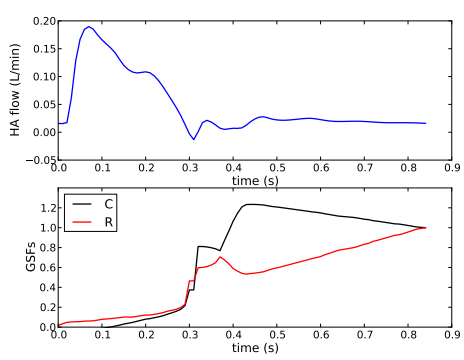

(b) After $75 \%$ hepatectomy

Figure 12: Flow rate GSF of hepatic arterial trees for the resistance and the capacitance

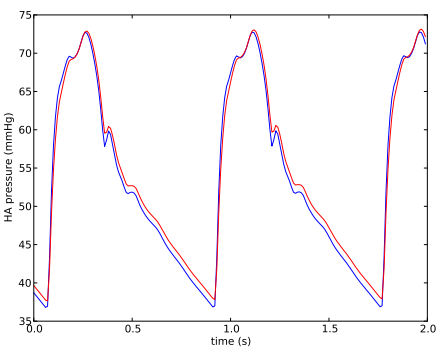

(a) Hepatic artery simulated pressure

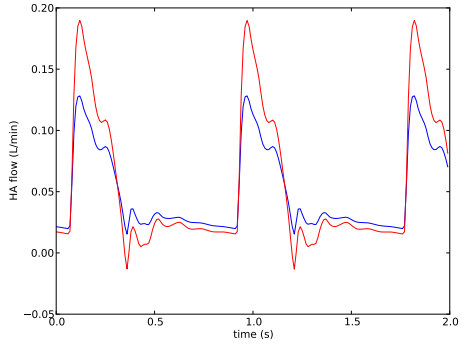

(b) Hepatic artery simulated flow

Figure 13: Simulated pressure and flow in the hepatic artery, with reduced capacitance and increased resistance in each of the 3 lobes (blue) and with previous parameters after the simulated $75 \%$ hepatectomy (red). 
changed, due to mass proportionality assumptions.

In summary, the modeling choices linking the liver resistances and capacitances to the mass and to the lobar structure of the liver allowed us to reproduce the changes in the experimentally observed signals. Thus, the study and reproduction of hepatectomy with a model enable us to better understand experimental observations and propose a novel link between architecture and flow. Monitoring waveform changes during post-hepatectomy regeneration could thus be a surrogate for the underlying architectural changes, which are currently not possible to non-invasively quantify.

\section{Conclusions}

In this work, the kinetic scheme, mainly used for the Saint-Venant equations in the literature, was successfully adapted to blood flow models. This scheme proved to have a very good behavior for arterial and venous benchmark tests. In particular, its theoretical properties of positivity make it especially well adapted to simulate collapsible vessels.

The scheme was then used to simulate complex behaviors occurring during liver surgeries. First, an idealized test representing the unclamping of the portal vein was proposed. Then the effects of partial hepatectomies on the hepatic artery pressure and flow waveforms were studied with a 1D-0D closedloop model. Interestingly, the changes observed experimentally on pigs were correctly captured for different percentages of hepatectomy. To the best of our knowledge, these experimental observations were never reported before in the literature. The capability of the model to represent this complex behavior allowed us to propose possible explanations of the observed phenomenon.

Future work will be devoted to a finer characterization of the change of the liver architecture during hepatectomy, and to the adaptation of the model to humans. 


\section{Acknowledgements}

This material is based upon work supported by the French National Agency for Research ANR-13-TECS-0006 iFLOW. The authors gratefully acknowledge Dr. Damiano Lombardi for assistance with implementation of the 1D models, and Dr. Jacques Sainte-Marie for his expertise in kinetic schemes. The authors are grateful to the INRA Plateforme CIRE (Nouzilly, France) staff for their technical assistance in surgeries and imaging, and to Mylène Wartenberg for assistance in taking measurements.

\section{References}

[1] S. Truant, O. Oberlin, G. Sergent, G. Lebuffe, L. Gambiez, O. Ernst, F.-R. Pruvot, Remnant liver volume to body weight ratio $0.5 \%$ : a new cut-off to estimate postoperative risks after extended resection in noncirrhotic liver, Journal of the American College of Surgeons 204 (1) (2007) 22-33.

[2] H. Boxenbaum, Interspecies variation in liver weight, hepatic blood flow, and antipyrine intrinsic clearance: extrapolation of data to benzodiazepines and phenytoin, Journal of pharmacokinetics and biopharmaceutics 8 (2) (1980) 165-176.

[3] W. W. Lautt, Regulatory processes interacting to maintain hepatic blood flow constancy: vascular compliance, hepatic arterial buffer response, hepatorenal reflex, liver regeneration, escape from vasoconstriction, Hepatology Research 37 (11) (2007) 891-903.

[4] T. J. Hughes, J. Lubliner, On the one-dimensional theory of blood flow in the larger vessels, Mathematical Biosciences 18 (1) (1973) 161-170.

[5] C. Guiot, P. Pianta, C. Cancelli, T. Pedley, Prediction of coronary blood flow with a numerical model based on collapsible tube dynamics, American Journal of Physiology-Heart and Circulatory Physiology 258 (5) (1990) H1606-H1614. 
[6] M. S. Olufsen, Structured tree outflow condition for blood flow in larger systemic arteries, Am J Physiol 276 (1 Pt 2) (1999) H257-68.

[7] L. Formaggia, D. Lamponi, A. Quarteroni, One-dimensional models for blood flow in arteries, Journal of engineering mathematics 47 (3-4) (2003) 251-276.

[8] S. Sherwin, L. Formaggia, J. Peiro, V. Franke, Computational modelling of 1D blood flow with variable mechanical properties and its application to the simulation of wave propagation in the human arterial system, International Journal for Numerical Methods in Fluids 43 (6-7) (2003) 673-700.

[9] I. E. Vignon, C. A. Taylor, Outflow boundary conditions for onedimensional finite element modeling of blood flow and pressure waves in arteries, Wave Motion 39 (4) (2004) 361-374.

[10] K. S. Matthys, J. Alastruey, J. Peiró, A. W. Khir, P. Segers, P. R. Verdonck, K. H. Parker, S. J. Sherwin, Pulse wave propagation in a model human arterial network: assessment of 1-D numerical simulations against in vitro measurements, Journal of biomechanics 40 (15) (2007) 3476-3486.

[11] J. Alastruey, A. W. Khir, K. S. Matthys, P. Segers, S. J. Sherwin, P. R. Verdonck, K. H. Parker, J. Peiró, Pulse wave propagation in a model human arterial network: assessment of 1-D visco-elastic simulations against in vitro measurements, Journal of biomechanics 44 (12) (2011) 2250-2258.

[12] X. Wang, O. Delestre, J.-M. Fullana, M. Saito, Y. Ikenaga, M. Matsukawa, P.-Y. Lagrée, Comparing different numerical methods for solving arterial 1D flows in networks, Computer Methods in Biomechanics and Biomedical Engineering 15 (sup1) (2012) 61-62.

[13] J. Keijsers, C. Leguy, W. Huberts, A. Narracott, J. Rittweger, F. van de Vosse, A 1D pulse wave propagation model of the hemodynamics of calf muscle pump function, International journal for numerical methods in biomedical engineering 31 (7). 
[14] L. Aslanidou, B. Trachet, P. Reymond, R. A. Fraga-Silva, P. Segers, N. Stergiopulos, A 1D model of the arterial circulation in mice., Altex 33 (1) (2016) 13.

[15] L. Formaggia, A. Quarteroni, A. Veneziani, Cardiovascular Mathematics Modeling and simulation of the circulatory system, Vol. 1, Springer, 2009.

[16] A. H. Shapiro, Steady flow in collapsible tubes, Journal of Biomechanical Engineering 99 (3) (1977) 126-147.

[17] T. J. Pedley, B. S. Brook, R. S. Seymour, Blood pressure and flow rate in the giraffe jugular vein, Philos Trans R Soc Lond B Biol Sci 351 (1342) (1996) 855-66. doi:10.1098/rstb.1996.0080.

[18] B. S. Brook, T. J. Pedley, A model for time-dependent flow in (giraffe jugular) veins: uniform tube properties, J Biomech 35 (1) (2002) 95-107.

[19] L. O. Müller, G. I. Montecinos, E. F. Toro, Some issues in modelling venous haemodynamics, Numerical Methods for Hyperbolic Equations: Theory and Applications. An international conference to honour Professor EF Toro (2012) 347-354.

[20] E. Boileau, P. Nithiarasu, P. J. Blanco, L. O. Müller, F. E. Fossan, L. R. Hellevik, W. P. Donders, W. Huberts, M. Willemet, J. Alastruey, A benchmark study of numerical schemes for one-dimensional arterial blood flow modelling, Int J Numer Method Biomed Eng 31 (10). doi:10.1002/cnm.2732.

[21] E. Marchandise, P. Flaud, Accurate modelling of unsteady flows in collapsible tubes, Computer Methods in Biomechanics and Biomedical Engineering 13 (2) (2010) 279-290.

[22] E. Audusse, M.-O. Bristeau, B. Perthame, Kinetic Schemes for SaintVenant Equations with Source Terms on Unstructured Grids, Research Report RR-3989, INRIA, projet M3N (2000).

URL https://hal.inria.fr/inria-00072657 
[23] O. Delestre, P.-Y. Lagrée, A 'well-balanced' finite volume scheme for blood flow simulation, International Journal for Numerical Methods in Fluids 72 (2) (2013) 177-205.

[24] B. Perthame, Kinetic formulation of conservation laws, Vol. 21, Oxford University Press, 2002.

[25] E. Audusse, F. Bouchut, M.-O. Bristeau, R. Klein, B. Perthame, A fast and stable well-balanced scheme with hydrostatic reconstruction for shallow water flows, SIAM Journal on Scientific Computing 25 (6) (2004) 20502065.

[26] G. I. Montecinos, L. O. Müller, E. F. Toro, Hyperbolic reformulation of a 1d viscoelastic blood flow model and ader finite volume schemes, Journal of Computational Physics 266 (2014) 101-123.

[27] C. Debbaut, D. De Wilde, C. Casteleyn, P. Cornillie, D. Van Loo, L. Van Hoorebeke, D. Monbaliu, Y.-D. Fan, P. Segers, Modeling the impact of partial hepatectomy on the hepatic hemodynamics using a rat model, Biomedical Engineering, IEEE Transactions on 59 (12) (2012) 3293-3303.

[28] H. Ho, K. Sorrell, A. Bartlett, P. Hunter, Blood flow simulation for the liver after a virtual right lobe hepatectomy, in: Medical Image Computing and Computer-Assisted Intervention-MICCAI 2012, Springer, 2012, pp. $525-532$.

[29] P. J. Blanco, R. A. Feijóo, A dimensionally-heterogeneous closed-loop model for the cardiovascular system and its applications, Med Eng Phys 35 (5) (2013) 652-67. doi:10.1016/j.medengphy.2012.07.011.

[30] F. Liang, S. Takagi, R. Himeno, H. Liu, Multi-scale modeling of the human cardiovascular system with applications to aortic valvular and arterial stenoses, Medical \& biological engineering \& computing 47 (7) (2009) 743755 . 
[31] J. P. Mynard, J. J. Smolich, One-dimensional haemodynamic modeling and wave dynamics in the entire adult circulation, Annals of biomedical engineering 43 (6) (2015) 1443-1460.

[32] L. O. Müller, E. F. Toro, A global multiscale mathematical model for the human circulation with emphasis on the venous system, International journal for numerical methods in biomedical engineering 30 (7) (2014) 681-725.

[33] B. Lantz, J. Foerster, D. Link, J. Holcroft, Regional distribution of cardiac output: normal values in man determined by video dilution technique, American Journal of Roentgenology 137 (5) (1981) 903-907.

[34] J. K. Raines, M. Y. Jaffrin, A. H. Shapiro, A computer simulation of arterial dynamics in the human leg, Journal of biomechanics 7 (1) (1974) 77-91.

[35] I. E. Vignon-Clementel, C. A. Figueroa, K. E. Jansen, C. A. Taylor, Outflow boundary conditions for 3D simulations of non-periodic blood flow and pressure fields in deformable arteries, Computer Methods in Biomechanics and Biomedical Engineering 13 (2010) 625-640. doi:10.1080/10255840903413565.

URL <Go to ISI > //WOS: 000281851500012

[36] J. F. LaDisa, C. Alberto Figueroa, I. E. Vignon-Clementel, H. Jin Kim, N. Xiao, L. M. Ellwein, F. P. Chan, J. A. Feinstein, C. A. Taylor, Computational Simulations for Aortic Coarctation: Representative Results From a Sampling of Patients, Journal of Biomechanical Engineering 133 (2011) 091008. doi:10.1115/1.4004996.

[37] P. Segers, N. Stergiopulos, N. Westerhof, P. Wouters, P. Kolh, P. Verdonck, Systemic and pulmonary hemodynamics assessed with a lumped-parameter heart-arterial interaction model, Journal of Engineering Mathematics 47 (2003) 185-199.

[38] D. Lombardi, Inverse problems in 1d hemodynamics on systemic networks: 
a sequential approach, Int J Numer Method Biomed Eng 30 (2) (2014) 160-79. doi:10.1002/cnm.2596.

[39] H. Suga, K. Sagawa, Instantaneous pressure-volume relationships and their ratio in the excised, supported canine left ventricle, Circulation research 35 (1) (1974) 117-126.

[40] G. Pennati, F. Migliavacca, G. Dubini, R. Pietrabissa, M. R. de Leval, A mathematical model of circulation in the presence of the bidirectional cavopulmonary anastomosis in children with a univentricular heart, Medical Engineering \& Physics 19 (3) (1997) 223-234.

[41] S. Pant, C. Corsini, C. Baker, T.-Y. Hsia, G. Pennati, I. Vignon-Clementel, Data assimilation and modelling of patient-specific single-ventricle physiology with and without valve regurgitation, Journal of Biomechanics doi:10.1016/j.jbiomech.2015.11.030.

[42] K. Thomaseth, C. Cobelli, Generalized sensitivity functions in physiological system identification, Annals of biomedical engineering 27 (5) (1999) 607616.

[43] P. Bai, H. T. Banks, S. Dediu, A. Y. Govan, M. Last, A. L. Lloyd, H. K. Nguyen, M. S. Olufsen, G. Rempala, B. D. Slenning, Stochastic and deterministic models for agricultural production networks, Math Biosci Eng 4 (3) (2007) 373-402.

[44] H. Miao, X. Xia, A. S. Perelson, H. Wu, On identifiability of nonlinear ode models and applications in viral dynamics, SIAM Rev Soc Ind Appl Math 53 (1) (2011) 3-39. doi:10.1137/090757009.

[45] S. Pant, B. Fabrèges, J.-F. Gerbeau, I. E. Vignon-Clementel, A methodological paradigm for patient-specific multi-scale cfd simulations: from clinical measurements to parameter estimates for individual analysis, Int $\mathrm{J} \mathrm{Nu}$ mer Method Biomed Eng 30 (12) (2014) 1614-48. doi:10.1002/cnm.2692. 


\begin{tabular}{ccccc}
\hline Heart chamber & Right atrium & Right ventricle & Left atrium & Left ventricle \\
\hline$E_{a}\left(\mathrm{dyn} / \mathrm{cm}^{5}\right)$ & 80 & 750 & 200 & 1600 \\
$E_{b}\left(\mathrm{dyn} / \mathrm{cm}^{5}\right)$ & 110 & 100 & 400 & 350 \\
$T_{c}(\mathrm{~s})$ & 0.145 & 0.289 & 0.145 & 0.289 \\
$T_{r}(\mathrm{~s})$ & 0.145 & 0.128 & 0.145 & 0.128 \\
$t_{c}(\mathrm{~s})$ & 0.68 & - & 0.68 & - \\
$t_{r}(\mathrm{~s})$ & 0.824 & - & 0.824 & - \\
$V_{0}\left(\mathrm{~cm}^{3}\right)$ & 4 & 10 & 4 & 5 \\
& $R_{p}\left(\right.$ dyn.s $\left./ \mathrm{cm}^{5}\right)$ & $R_{d}\left(\right.$ dyn.s $\left./ \mathrm{cm}^{5}\right)$ & $\mathrm{C}\left(\mathrm{cm}^{5} / \mathrm{dyn}\right)$ & \\
Lungs & 53 & 53 & 0.03 & \\
Vena cava & 10 & 10 & 0.004 & \\
\hline
\end{tabular}

Table 1: Parameter for heart, lungs and vena cava OD models. $E_{a}$ is the contraction function amplitude, $E_{b}$ the contraction function baseline, $T_{c}$ is the duration of contraction, $T_{r}$ is the duration of relaxation, $t_{c}$ and $t_{r}$ are the times when the atria begin to contract and relax, respectively and $V_{0}$ is the unstressed volume of the chamber. $R_{p}$ and $R_{d}$ are the proximal and distal resistances of the RCR model and $\mathrm{C}$ is the capacitance.

\section{Appendix}

\subsection{Parameter and error tables}

In this appendix, tables of the 1D and 0D model parameters are summarized, along with precise errors for benchmark test results as referred to in the text.

\subsection{Generalized sensitivity functions}

For those unfamiliar with the GSFs, we recall their definition [42, 45]. Consider the model for the state vector $\mathbf{x}=\left[x_{1}, x_{2}, \ldots, x_{L}\right]$ :

$$
\dot{x_{i}}(t)=f_{i}(t, \mathbf{x}, \boldsymbol{\theta}) \quad i=1,2, \ldots, L
$$

where $\boldsymbol{\theta}=\left[\theta_{1}, \theta_{2}, \ldots, \theta_{P}\right]$ is the model parameters vector and the dynamic model is represented with functions $f_{i}$. The observation vector $\boldsymbol{z}=\left[z_{1}, \ldots, z_{M}\right]$, can be written as :

$$
\boldsymbol{z}\left(t_{n}\right)=\mathbf{h}\left(t_{n}, \boldsymbol{\theta}\right)+\boldsymbol{\epsilon}\left(t_{n}\right) \quad n=1,2, . ., N \quad \text { with } \mathbf{h}(t, \boldsymbol{\theta})=H(\mathbf{x}(t, \boldsymbol{\theta}))
$$




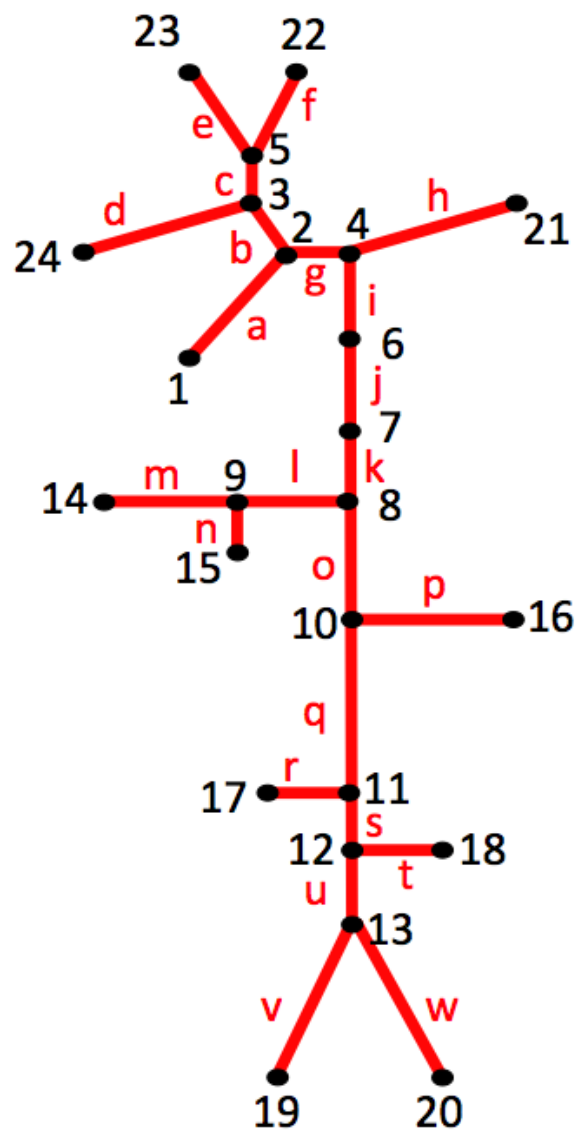

Figure 14: 1D arteries node number and arteries id; see Table 2 for their parameter values. 


\begin{tabular}{|c|c|c|c|c|c|}
\hline id & Name & $\mathrm{l}(\mathrm{cm})$ & $A_{0}\left(\mathrm{~cm}^{2}\right)$ & $\beta / \rho\left(\mathrm{cm} / \mathrm{s}^{2}\right)$ & $N_{e l}$ \\
\hline $\mathrm{a}$ & Ascending aorta & 3.5 & 2.54 & $2.0710^{5}$ & 35 \\
\hline $\mathrm{b}$ & Brachiocephalic trunk & 2.92 & 0.46 & $4.8810^{5}$ & 30 \\
\hline c & Aortic arch A & 0.36 & 0.39 & $5.3410^{5}$ & 5 \\
\hline d & Right subclavian & 8.5 & 0.20 & $7.9610^{5}$ & 85 \\
\hline $\mathrm{e}$ & Right common carotid & 11.8 & 0.12 & $1.2410^{6}$ & 120 \\
\hline $\mathrm{f}$ & Left common carotid & 11.9 & 0.15 & $9.7910^{5}$ & 120 \\
\hline $\mathrm{g}$ & Aortic arch B & 0.68 & 2.30 & $2.1710^{5}$ & 7 \\
\hline $\mathrm{h}$ & Left Subclavian & 12 & 0.31 & $6.0110^{5}$ & 120 \\
\hline i & Thoracic aorta A & 9.1 & 2.06 & $2.3010^{5}$ & 91 \\
\hline $\mathrm{j}$ & Thoracic aorta B & 9.5 & 1.43 & $2.7510^{5}$ & 95 \\
\hline $\mathrm{k}$ & Thoracic aorta $\mathrm{C}$ & 9.5 & 0.81 & $3.6610^{5}$ & 95 \\
\hline 1 & Celiac trunk & 0.66 & 0.29 & $6.2210^{5}$ & 7 \\
\hline $\mathrm{m}$ & Hepatic artery & 5 & 0.10 & $1.4510^{6}$ & 50 \\
\hline $\mathrm{n}$ & Splenic artery & 12.8 & 0.10 & $1.4510^{6}$ & 130 \\
\hline o & Abdominal aorta A & 1.7 & 0.80 & $3.6910^{5}$ & 17 \\
\hline $\mathrm{p}$ & Mesenteric artery & 3 & 0.36 & $5.5710^{5}$ & 30 \\
\hline q & Abdominal aorta B & 3.55 & 0.80 & $3.6910^{5}$ & 36 \\
\hline $\mathrm{r}$ & Right Renal & 3.65 & 0.18 & $8.6310^{5}$ & 37 \\
\hline $\mathrm{s}$ & Abdominal aorta $\mathrm{C}$ & 0.5 & 0.78 & $3.7210^{5}$ & 5 \\
\hline $\mathrm{t}$ & Left renal & 1.37 & 0.24 & $6.9510^{5}$ & 14 \\
\hline $\mathrm{u}$ & Abdominal aorta D & 8 & 0.57 & $4.3610^{5}$ & 80 \\
\hline $\mathrm{v}$ & Right iliac & 2.9 & 0.29 & $6.3310^{5}$ & 29 \\
\hline $\mathrm{w}$ & Left iliac & 2.8 & 0.34 & $5.7410^{5}$ & 28 \\
\hline
\end{tabular}

Table 2: Parameters for the 1D vessels of the pig cardiovascular model. The first column contains the id number of the artery (Figure 14), the second the name of the artery, the third 1 its length in $\mathrm{cm}$, the fourth its cross-section area $A_{0}$ in $\mathrm{cm}^{2}$ from CT-scan, the fifth is the vessel elasticity coefficient $\beta$ (defined in equation (14)) divided by the fluid density $\rho$, and the last one the number of elements used to discretize the vessel. 


\begin{tabular}{ccccc}
\hline id & out & $R_{p}\left(\right.$ dyn.s $\left./ \mathrm{cm}^{5}\right)$ & $R_{d}\left(\right.$ dyn.s $\left./ \mathrm{cm}^{5}\right)$ & $\mathrm{C}\left(\mathrm{cm}^{5} / \mathrm{dyn}\right)$ \\
\hline $\mathrm{n}$ & 15 & 953 & 8584 & $2.010^{-5}$ \\
$\mathrm{p}$ & 16 & 864 & 7780 & $4.010^{-5}$ \\
$\mathrm{r}$ & 17 & 1189 & 10705 & $2.010^{-5}$ \\
$\mathrm{t}$ & 18 & 1196 & 10762 & $2.010^{-5}$ \\
$\mathrm{v}$ & 19 & 1655 & 1490 & $8.010^{-5}$ \\
$\mathrm{w}$ & 20 & 165 & 14907 & $8.010^{-5}$ \\
$\mathrm{~h}$ & 21 & 1069 & 9622 & $6.010^{-5}$ \\
$\mathrm{f}$ & 22 & 1288 & 11592 & $4.010^{-5}$ \\
$\mathrm{e}$ & 23 & 1311 & 11806 & $4.010^{-5}$ \\
$\mathrm{~d}$ & 24 & 1063 & 9566 & $6.010^{-5}$ \\
\hline
\end{tabular}

Table 3: Parameters for the outlets RCR models. The first column contains the id of the artery, the second node id, the third $R_{p}$ the proximal resistance, the fourth $R_{d}$ the distal resistance, and the last one the capacitance.

\begin{tabular}{cccc}
\hline Liver lobes & Right lobe & Middle lobe & Left lobe \\
\hline Mass $(\mathrm{g})$ & 250 & 500 & 180 \\
$R_{p} / R_{\text {tot }}$ arterial tree & 0.1 & 0.5 & 0.1 \\
\hline & Arterial tree & Portal vein tree & Tissue + Hepatic veins \\
\hline Mass resistance $\left(\right.$ g.dyn.s $\left./ \mathrm{cm}^{5}\right)$ & $1.910^{7}$ & $1.8610^{5}$ & $7.4410^{4}$ \\
Mass capacitance $\left(\mathrm{cm}^{5} / \mathrm{dyn} / \mathrm{g}\right)$ & $3.010^{-8}$ & - & $1.510^{-5}$ \\
\hline & Hepatectomy & \\
$75 \%$ clamping time $T_{75}(\mathrm{~s})$ & parameters & & \\
$90 \%$ clamping time $T_{90}(\mathrm{~s})$ & 16 & \\
$r(\%)$ & 35 & \\
\hline
\end{tabular}

Table 4: Liver OD model parameters. First the different lobe masses are given and the ratio between proximal and total resistances in each lobe for the hepatic artery RCR model. Then, mass resistance and mass capacitance are given for hepatic artery tree, portal vein tree, tissue and hepatic vein tree, followed by the clamping parameters. $r$ is the right lobe resected mass \%. 


\begin{tabular}{cc}
\hline Time step $(\mathrm{s})$ & $10^{-5}$ \\
Mesh size $(\mathrm{cm})$ & 0.1 \\
\hline initial pressure $p(x, t=0)\left(\right.$ dyn.cm $\left.^{-2}\right)$ & $6.010^{4}$ \\
initial velocity $u(x, t=0)(\mathrm{cm} / \mathrm{s})$ & 5 \\
$P_{0}\left(\right.$ dyn.cm $\left.^{-2}\right)$ & $6.610^{4}$ \\
$K_{f}\left(\mathrm{~cm}^{2} / \mathrm{s}\right)$ & 3 \\
$\rho\left(\mathrm{g} \cdot \mathrm{cm}^{-3}\right)$ & 1.05 \\
\hline
\end{tabular}

Table 5: 1D-0D closed-loop simulation parameters, time and space discretization parameters, initial conditions for $1 \mathrm{D}$ part, $P_{0}$, friction parameter and fluid density values.

\begin{tabular}{|c|c|}
\hline Properties & Values \\
\hline Length $\mathrm{L}$ & $10 \mathrm{~m}$ \\
\hline Cross-section area $A_{0}$ & $\pi \mathrm{cm}^{2}$ \\
\hline Initial velocity $\mathrm{u}(\mathrm{x}, 0)$ & $0 \mathrm{~cm} / \mathrm{s}$ \\
\hline Initial pressure $\mathrm{P}(\mathrm{x}, 0)$ & 0 dyn.cm \\
\hline Pressure $P_{0}$ & 0 dyn.cm \\
\hline Wall thickness $h$ & $0.15 \mathrm{~cm}$ \\
\hline Young's modulus $\mathrm{E}$ & $4.010^{5}$ dyn.cm ${ }^{-2}$ \\
\hline Elasticity parameter $\beta$ & $4.51510^{5}$ dyn.cm ${ }^{-3}$ \\
\hline Blood mass density $\rho$ & 1.05 g.cm ${ }^{-3}$ \\
\hline Blood viscosity $\mu$ & 0 or 0.04 dyn.cm ${ }^{-2}$ \\
\hline Friction term $K_{f}$ & $22 \pi \frac{\mu}{\rho}$ \\
\hline
\end{tabular}

Table 6: Parameters for the single pulse propagation benchmark test case from [20]. 


\begin{tabular}{|c|cc|cc|cc|cc|}
\hline & \multicolumn{2}{|c|}{ Case 1} & \multicolumn{2}{c|}{ Case 2} & \multicolumn{2}{c|}{ Case 3} & \multicolumn{2}{c|}{ Case 4} \\
\hline instants & $\mathcal{E}_{l^{2}}$ & $\mathcal{E}_{\infty}$ & $\mathcal{E}_{l^{2}}$ & $\mathcal{E}_{\infty}$ & $\mathcal{E}_{l^{2}}$ & $\mathcal{E}_{\infty}$ & $\mathcal{E}_{l^{2}}$ & $\mathcal{E}_{\infty}$ \\
$\mathrm{t}=0.1 \mathrm{~s}$ & 0.036 & 0.043 & 0.017 & 0.007 & 0.007 & 0.012 & 0.007 & 0.012 \\
$\mathrm{t}=0.3 \mathrm{~s}$ & 0.145 & 0.166 & 0.060 & 0.024 & 0.028 & 0.047 & 0.029 & 0.047 \\
$\mathrm{t}=0.5 \mathrm{~s}$ & 0.230 & 0.251 & 0.104 & 0.040 & 0.049 & 0.073 & 0.049 & 0.077 \\
$\mathrm{t}=0.7 \mathrm{~s}$ & 0.292 & 0.314 & 0.147 & 0.055 & 0.067 & 0.099 & 0.067 & 0.101 \\
$\mathrm{t}=0.9 \mathrm{~s}$ & 0.343 & 0.364 & 0.189 & 0.069 & 0.083 & 0.121 & 0.083 & 0.122 \\
$\mathrm{t}=1.1 \mathrm{~s}$ & 0.384 & 0.402 & 0.229 & 0.083 & 0.099 & 0.140 & 0.097 & 0.141 \\
$\mathrm{t}=1.3 \mathrm{~s}$ & 0.419 & 0.438 & 0.267 & 0.096 & 0.111 & 0.158 & 0.111 & 0.158 \\
$\mathrm{t}=1.5 \mathrm{~s}$ & 0.448 & 0.465 & 0.305 & 0.109 & 0.124 & 0.173 & 0.122 & 0.174 \\
\hline
\end{tabular}

Table 7: Normalized errors for the single pulse propagation test case; for cases 1,2,3 friction is neglected and case 4 is the viscous blood case. The normalized errors are defined by $\mathcal{E}_{l^{2}}=$ $\left\|X_{k i n}-X_{S T M}\right\|_{l^{2}} /\left\|X_{S T M}\right\|_{l^{2}}$ and $\mathcal{E}_{\infty}=\left\|X_{k i n}-X_{S T M}\right\|_{\infty} /\left\|X_{S T M}\right\|_{\infty}$ where $X_{S T M}$ is the solution with the 1D STM scheme from [20] and $X_{k i n}$ is the solution obtained with the kinetic scheme. Case 1 presents the results of the first order kinetic scheme with $\Delta x=0.1 \mathrm{~cm}$, and $\Delta t=1.010^{-4} \mathrm{~s}$, case 2 of the first order kinetic scheme with $\Delta x=0.01 \mathrm{~cm}$, and $\Delta t=1.010^{-5} \mathrm{~s}$, case 3 of the first order in time and second order in space kinetic scheme with $\Delta x=0.1 \mathrm{~cm}$, and $\Delta t=1.010^{-5} \mathrm{~s}$, finally case 4 of the first order in time and second order in space kinetic scheme with $\Delta x=0.1 \mathrm{~cm}$ and $\Delta t=1.010^{-5} \mathrm{~s}$ with a non-zero friction term. 


\begin{tabular}{|c|c|c|}
\hline Properties & Aorta & Iliac \\
\hline Length L & $8.6 \mathrm{~cm}$ & $8.5 \mathrm{~cm}$ \\
\hline Cross-section area $A_{0}$ & $2.3235 \mathrm{~cm}^{2}$ & $1.131 \mathrm{~cm}^{2}$ \\
\hline Initial velocity $\mathrm{u}(\mathrm{x}, 0)$ & $0 \mathrm{~cm} / \mathrm{s}$ & $0 \mathrm{~cm} / \mathrm{s}$ \\
\hline Initial pressure $\mathrm{P}(\mathrm{x}, 0)$ & 0 dyn.cm ${ }^{-2}$ & 0 dyn.. $\mathrm{cm}^{-2}$ \\
\hline Pressure $P_{0}$ & $9.4610^{4}$ dyn.cm ${ }^{-2}$ & $9.4610^{4}$ dyn.cm ${ }^{-2}$ \\
\hline Wall thickness $h$ & $0.1032 \mathrm{~cm}$ & $0.072 \mathrm{~cm}$ \\
\hline Young's modulus E & $5.010^{5} \mathrm{dyn}_{\mathrm{cm}^{-2}}$ & $7.010^{5}$ dyn $\mathrm{cm}^{-2}$ \\
\hline Elasticity parameter $\beta$ & $4.67110^{5}$ dyn.cm ${ }^{-3}$ & $9.372810^{5}$ dyn.. $\mathrm{cm}^{-3}$ \\
\hline Blood mass density $\rho$ & 1.06 g.cm ${ }^{-3}$ & 1.06 g.cm ${ }^{-3}$ \\
\hline Blood viscosity $\mu$ & 0.04 dyn.cm ${ }^{-2}$ & 0.04 dyn.cm ${ }^{-2}$ \\
\hline Friction term $K_{f}$ & $22 \pi \frac{\mu}{\rho}$ & $22 \pi \frac{\mu}{\rho}$ \\
\hline Windkessel proximal resistance $R_{p}$ & - & $6.812310^{2}$ dyn.s.cm ${ }^{-5}$ \\
\hline Windkessel distal resistance $R_{d}$ & - & $3.101310^{4}$ dyn.s.cm ${ }^{-5}$ \\
\hline Windkessel capacitance $C$ & - & $3.666410^{-5} \mathrm{~cm}^{5} \cdot \mathrm{dyn}^{-1}$ \\
\hline
\end{tabular}

Table 8: Parameters for the aortic bifurcation benchmark test case from [20]. 


\begin{tabular}{|l|c|c|c|}
\hline Error & Midpoint Aorta & End point Aorta & Midpoint Iliac \\
\hline $\mathcal{E}_{P}^{R M S}$ & 0.39 & 0.42 & 0.45 \\
$\mathcal{E}_{Q}^{R M S}$ & 0.93 & 1.17 & 0.53 \\
$\mathcal{E}_{\Delta r}^{R M S}$ & 2.41 & 3.99 & 4.21 \\
$\mathcal{E}_{P}^{M A X}$ & 0.67 & 0.78 & 0.9 \\
$\mathcal{E}_{Q}^{M A X}$ & 2.81 & 3.64 & 2.09 \\
$\mathcal{E}_{\Delta r}^{M A X}$ & 3.87 & 6.74 & 7.25 \\
$\mathcal{E}_{P}^{S Y S}$ & -0.46 & -0.64 & -0.77 \\
$\mathcal{E}_{Q}^{S Y S}$ & -2.51 & -3.51 & -1.56 \\
$\mathcal{E}_{\Delta r}^{S Y S}$ & -3.72 & -6.61 & -7.03 \\
$\mathcal{E}_{P}^{D I A S}$ & 0.4 & 0.45 & 0.46 \\
$\mathcal{E}_{Q}^{D I A S}$ & 1.15 & 1.74 & 1.05 \\
$\mathcal{E}_{\Delta r}^{D I A S}$ & -1.42 & -1.95 & -2.37 \\
\hline
\end{tabular}

Table 9: Error for the aortic bifurcation test case with respect to 3D solution in percent as defined in [20]. 
where $H$ is the observation operator, $\mathbf{h}$ represents the noise-free observation vector and the vector $\boldsymbol{\epsilon}\left(t_{n}\right)$ is the noise on measurements at time $t_{n}$. The noise vectors are assumed to be independent for all measurement times. Moreover, all components of the noise vector are assumed independent with zero mean and $\sigma_{i}^{2}\left(t_{n}\right)$ variance associated with measurement $z_{i}\left(t_{n}\right)$. The generalized sensitivity function for the parameter $\theta_{k}$ is defined by :

$$
g_{k}\left(t_{n}\right)=\sum_{i=1}^{n} \sum_{j=1}^{M}\left(\frac{1}{\sigma_{j}^{2}\left(t_{j}\right)}\left(\mathcal{M}^{-1} \nabla_{\theta} h_{j}\left(t_{i}, \boldsymbol{\theta}_{\mathbf{0}}\right)\right)_{k}\left(\nabla_{\theta} h_{j}\left(t_{i}, \boldsymbol{\theta}_{\mathbf{0}}\right)\right)_{k}\right)
$$

The sensitivity is computed around a reference parameter vector $\boldsymbol{\theta}_{\mathbf{0}}$. The matrix $\mathcal{M}$ denotes the Fisher information matrix, defined as

$$
\mathcal{M}=\sum_{i=1}^{N} \sum_{j=1}^{M} \frac{1}{\sigma_{j}^{2}\left(t_{j}\right)}\left(\nabla_{\theta} h_{j}\left(t_{i}, \boldsymbol{\theta}_{\mathbf{0}}\right)\right)\left(\nabla_{\theta} h_{j}\left(t_{i}, \boldsymbol{\theta}_{\mathbf{0}}\right)\right)^{T}
$$

